\title{
FAMILIE, VERHÄLTNISSE ZWISCHEN EHEGATTEN UND NICHTEHELICHE LEBENSGEMEINSCHAFTEN IM POLNISCHEN UND ISLAMISCHEN RECHT ${ }^{1}$
}

\begin{abstract}
Zusammenfassung
In der 32. Sitzung des Sejms der gegenwärtigen Legislaturperiode hat die Kammer in der ersten Lesung fünf Gesetzentwürfe über die breit gefasste Regulierung von Lebenspartnerschaften abgelehnt. Art. 18 der Verfassung der Republik Polen sieht vor: „Die Ehe als Verbindung von Frau und Mann, Familie, Mutterschaft und das Elternrecht stehen unter dem Schutz und der Obhut der Republik Polen". Dieser Artikel ordnet den Staat an, solche Maßnahmen vorzunehmen, die die Bindung zwischen den die Familie bildenden Personen verstärken. Art. 18 der Verfassung, indem er den öffentlichen Gewaltorganen der Republik Polen auferlegt, Ehe und Familie unter Schutz und Obhut zu stellen, impliziert das Verbot, durch diese Organe Maßnahmen zu ergreifen, die das Ziel haben, für die tatsächlich bestehenden Emotional- und Vermögensbeziehung von zwei Personen, die eine nichteheliche Form der Lebensgemeinschaft darstellt, solche Regelungen zu schaffen, die sie in Rechtsgestalt an die Ehe bezüglich der Bestimmung von Rechten und Pflichten der Personen, die sich in dieser Beziehung befinden sowie der Frage ihrer Schließung oder Auflösung, anpassen oder sie dieser gleichsetzen.

Die Scharia - das islamische Recht - sieht vor, dass die aus einem Mann und einer Frau bestehende Familie die Grundlage der Gesellschaft bildet, weil die Ehe die einzige berechtigte, legale Art der Befriedigung des sexuellen Triebes zwischen Mann und Frau ist. Das Wesen des islamischen Familienrechts ist eine vollständige Kontrolle und Regulierung des Familienlebens. Dieses Ziel schwebt auch dem ehelichen Vertrag vor, der die Rechte und Pflichten der Parteien bestimmt, von denen nur eine (der Mann) an den Vertragsverhandlungen teilnimmt.
\end{abstract}

\section{Keyword}

die Ehe - die Familie - die Scharia - nikah - mahr

* Prof. dr dr h.c.; Universität in Wrocław.

** Prof. UWr dr habil.; Universität in Wrocław. 


\section{Polnisches ReCHT}

In der 32. Sitzung des Sejms der gegenwärtigen Legislaturperiode hat die Kammer in der ersten Lesung fünf Gesetzentwürfe über die breit gefasste Regulierung von Lebenspartnerschaften abgelehnt. Das waren: ein Gesetzentwurf über Lebenspartnerschaften (Drucksache Nr. 552), ein Gesetzentwurf - Einführungsgesetz zum Gesetz über Lebenspartnerschaften (Drucksache Nr. 553), ein Gesetzentwurf über Lebenspartnerschaften (Drucksache Nr. 554), ein Gesetzentwurf - Einführungsgesetz zum Gesetz über Lebenspartnerschaften (Drucksache Nr. 555), ein Gesetzentwurf über den Vertrag der Lebenspartnerschaft (Drucksache Nr. 825). Während der Debatte im Sejm und auch davor, in den der Sejmkanzlei überreichten Gutachten, hat die Verfassungsmäßigkeit aller dieser Gesetzentwürfe eine besondere Kontroverse hervorgerufen.

Art. 18 der Verfassung der Republik Polen sieht vor: „Die Ehe als Verbindung von Frau und Mann, Familie, Mutterschaft und das Elternrecht stehen unter dem Schutz und der Obhut der Republik Polen“. Aus der Hervorhebung der Rolle der Frau und des Mannes in einer Ehe durch den Verfassungsgeber folgt eine Stütze der Ehe auf ein Freiwilligkeitsprinzip und die Behandlung der Entscheidung über die Eheschließung als eine Realisierung der dem Menschen gewährten Freiheit. Das betrifft auch die Möglichkeit, die Ehe aufzulösen oder über Trennung ohne Auflösung des Ehebandes zu entscheiden. Daraus folgt auch die Gleichstellung der Vertreter der beiden Geschlechter im Bereich der ihnen zustehenden Rechte und Pflichten in der Ehe und in der Familie und auch gegenüber den Kindern.

Art. 18 befindet sich im ersten Kapitel, in dem die Ordnungsprinzipien der Republik Polen und das Wertesystem, auf das sich der polnische Staat stützt, bestimmt werden. Der Unterschied zwischen den Leitprinzipien und den sonstigen Verfassungsnormen hat nicht nur einen theoretischen

\footnotetext{
1 Nach unserer Beurteilung scheint es gerechtfertigt $\mathrm{zu}$ sein, den Begriff islamisches Familienrecht anzuwenden, weil die rechtlichen Vorschriften der vorliegenden Problematik in allen islamischen Ländern sehr ähnlich sind und sich auf die Scharia stützen.
} 
Charakter. Erstens hat er eine erhebliche praktische Bedeutung, weil ihre Änderung bestimmte Rechtswirkungen zur Folge hat. Die Verfassung sieht vor, dass es schwieriger ist, die Bestimmungen der Kapitel I, II und XI zu ändern. Zweitens bilden zwar alle Verfassungsnormen ein einheitliches System und gehören zu einer Rechtsquelle mit der höchsten Rechtskraft (Art. 8 der Verfassung der Republik Polen), was den Eindruck erweckt, dass alle Verfassungsnormen den gleichen normativen Charakter haben und dass man sie innerlich nicht differenzieren soll, aber nach der Absicht des Verfassungsgebers die Normen aus Kapitel I besonders zu behandeln sind. Obwohl alle Bestimmungen der Verfassung rechtlich bindend sind und im gleichen Ausmaß beachtet werden sollen, haben sie nicht die gleiche Bedeutung und die Verfassung ist (ähnlich wie sonst die Grundgesetze der Mehrheit der demokratischen Staaten) innerlich hierarchisiert. Das hat eine erhebliche Bedeutung sowohl im Anwendungsprozess als auch bei der Auslegung ihrer Normen. Das lässt z.B. zu, die Kollision von Verfassungsnormen aufzulösen. Dazu dient die Hervorhebung der Normen in Kapitel I, die einen besonders bedeutsamen Prinzipiencharakter haben. Diesen Normen sollte man den Vorrang vor den anderen Normen der Verfassung geben, weil sie die wichtigsten Inhalte der rechtlichen Ordnung ausdrücken und die übrigen Verfassungsbestimmungen im Verhältnis zu ihnen mehr oder weniger eine dienliche Rolle spielen und die detaillierten Verfassungsbestimmungen nach den fundamentalen Ordnungsprinzipien ausgelegt sein müssen und nicht getrennt von ihnen².

Unter Bezugnahme auf seine ständige Rechtsprechung hat der Verfassungsgerichtshof bezüglich des Art. 18 festgestellt: „Einerseits stellt diese Vorschrift eine Auslegungsdirektive der übrigen Verfassungsund Gesetzesnormen dar, die eine möglichst umfangreiche Realisierung der Prinzipien des Schutzes von Mutterschaft, Elternrecht und Ehe anordnet. Anderseits hat Art. 18 die Rolle einer Programmnorm. Das ist ein Element der vom Verfassungsgeber angenommenen Verfassungsaxiologie. Art. 18 der Verfassung befindet sich im ersten Kapitel, in dem

2 Vgl. SK 24/02, Orzecznictwo Trybunału Konstytucyjnego; Zbiór Urzędowy, Seria A [Rechtsprechung des Verfassungsgerichtshofs, Amtliche Sammlung, Serie A, OTK-A] 2003, Nr. 4, Pos. 33. 
die Ordnungsprinzipien der Republik Polen bestimmt werden. Dieser Artikel ordnet den Staat an, solche Maßnahmen vorzunehmen, die Bindung zwischen den die Familie bildenden Personen verstärken, u.a. die zwischen den Ehegatten sowie zwischen den Eltern und Kindern bestehenden Bindungen“3. Bei der Berufung auf die divergierenden Auffassungen im Schrifttum des Staatsrechts stellt der Verfassungsgerichtshof überdies fest, "dass ein einziges normatives Element, das sich aus dem Art. 18 der Verfassung entschlüsseln lässt, eine Festlegung des heterosexuellen Prinzips der Ehe ist ${ }^{\text {" }} 4$. Die besondere Rolle des Art. 18 für die Auslegung der anderen Verfassungsnormen betonend, stellt der Verfassungsgerichtshof fest: „Dass Art. 18 der Verfassung zu einem Kontrollmuster gemacht wurde, ist zweckmäßig, weil keine Vorschrift des zweiten Kapitels der Verfassung, das einen Katalog von verfassungsrechtlichen Rechten und Freiheiten enthält, eine angemessene Grundlage für die Ableitung der verfassungsrechtlichen subjektiven Rechte darstellt, die aus der Tatsache der Eheschließung folgen" 5 .

Der Staat, indem er die Ehe unterstützt, hat seit langem die Vorteile für die Gesellschaft aus dem Bestehen der formalisierten Beziehung der Frau und des Mannes, der stabilen und beständigen Beziehung wahrgenommen, in der sich die Ehegatten gegenseitig unterstützen, indem sie die Pflichten teilen und das gemeinsame Vermögen verwalten, sowie die Kinder betreuen, wenn solche aus ihrer Beziehung hervorgehen. Das wurde bei Sicherung der besonderen Position der Ehe in der Verfassung im Vergleich $\mathrm{zu}$ anderen möglichen Formen des Zusammenlebens von Menschen und durch die Ermunterung zur Eheschließung im Wege der Annahme von rechtlichen Lösungen gezeigt, die verschiedene Lebensaspekte der Einheiten und der Funktionen der Gesellschaft betreffen. Eine solche Auffassung der Ehe überwiegt

3 Urteil vom 11.05.2011, SK 11/09, Orzecznictwo Trybunału Konstytucyjnego; Zbiór Urzędowy, Seria A [Rechtsprechung des Verfassungsgerichtshofs, Amtliche Sammlung, Serie A, OTK-A] 2011, Nr. 4, Pos. 32.

4 Urteil vom 9.11.2010, SK 10/08, Orzecznictwo Trybunału Konstytucyjnego; Zbiór Urzędowy, Seria A [Rechtsprechung des Verfassungsgerichtshofs, Amtliche Sammlung, Serie A, OTK-A] 2010, Nr. 9, Pos. 99.

5 Fußnote 3. 
entschieden heutzutage, insbesondere im Kreis der Länder, in denen sich die Ordnung aus einer breit begriffenen europäischen Kultur herleiten lässt. Die Mehrheit von ihnen erkennt weder rechtliche Beziehungen der Personen gleichen Geschlechts noch nichteheliche Lebensgemeinschaften der Personen verschiedenen Geschlechts an. Die rechtlichen Bestimmungen (auf verschiedenem Niveau in der Hierarchie der Rechtsquellen), die unterschiedliche Lebenspartnerschaften definieren, treten in 30 modernen Staaten auf, und in 11 von ihnen wurde die Erweiterung der Institution Ehe auf die Personen gleichen Geschlechts ausgedehnt. Es ist bemerkenswert an dieser Stelle hinzuzufügen, dass in manchen Ländern oder in Bestandteilen der Bundesstaaten unter dem Einfluss der Diskussion zum Thema der verfassungsrechtlichen Auffassung der Ehe erst in letzten Jahren entschieden wurde, in die Grundgesetze eindeutige Rechtsformeln einzuführen, die dem Art. 18 der Verfassung der Republik Polen ähnlich sind. In diesem Zusammenhang ist hervorzuheben, dass die Europäische Menschenrechtskonvention die traditionelle Konzeption der Ehe und Familie als ein Verhältnis der Frau und des Mannes widerspiegelt und in ihrem Art. 12 das Recht garantiert, eine Ehe zu schließen und eine Familie zu gründen („Männer und Frauen im heiratsfähigen Alter haben das Recht, nach den innerstaatlichen Gesetzen, welche die Ausübung dieses Rechts regeln, eine Ehe einzugehen und eine Familie zu gründen“). Der Europäische Gerichtshof für Menschenrechte gibt in seiner Rechtsprechung $\mathrm{zu}$, dass der Schutz der Familie im traditionellen Sinn nach wie vor eines der Ziele der Konvention darstellt 6 .

Das traditionelle Konzept der Familie bringen auch die Akten des internationalen universalen Rechts zum Ausdruck, die sie als "natürliche und grundlegende Einheit der Gesellschaft" (Art. 16 Abs. 3 der Allgemeinen Erklärung der Menschenrechte, Art. 23 Abs. 1 Internationaler Pakt über bürgerliche und politische Rechte) bezeichnen. Sie behandeln die Ehe als eine Beziehung von Frau und Mann

\footnotetext{
6 Vgl. die angeführte Rechtsprechung in: L. Garlicki (Red.), Konwencja o Ochronie Praw Człowieka i Podstawowych Wolności, Tom I., Komentarz do art. 1-18 [Europäische Konvention zum Schutz der Menschenrechte und Grundfreiheiten, Band 1., Kommentar (Art. 1-18)], Warszawa: C.H. Beck 2010, S. 712-713.
} 
(Art. 16 Abs. 1 der Allgemeinen Erklärung der Menschenrechte, Art. 23 Abs. 2 Internationaler Pakt über bürgerliche und politische Rechte).

In der Rechtslehre wird auf die kulturelle, soziologische und naturrechtliche Begründung der Ehe hingewiesen. „Die Institution der Ehe ist also keine rein juristische Konstruktion, weil ihr Charakter starke empirische Elemente enthält, die sich auf die anderen Sozialwissenschaften, insbesondere auf die Soziologie, beziehen. Die Ehe als eine rechtliche Institution hat zugleich gewisse natürliche, biologische Eigenschaften, die sich empirisch verifizieren lassen, die nur der Ehe angemessen sind und keine andere institutionelle gesellschaftliche Beziehung verfügt über diese Merkmale. Aus rechtlicher Sicht dient die Institution der Ehe auch dem Schutz der Naturrechte der Ehegatten und der Naturrechte ihrer Kinder, also der unabhängig von dem Willen des Gesetzgebers bestehenden Rechte, der nur die Nuancen der rechtlichen Reglementierung dieser Rechte ausfeilen kann, jedoch ihnen nie einen besonderen Rechtsschutz verweigern darf"

Auf die angeführten Kultur-, Zivilisations- und Rechtswurzeln bezieht sich Art. 18 der Verfassung, in dem die Ehe eindeutig als ein Verhältnis von Frau und Mann bezeichnet wird. Die Anwendung des Singulars weist deutlich darauf hin, dass es um die Beziehung einer Frau und eines Mannes geht und die Ablehnung der Beziehungen der Personen gleichen Geschlechts, der polygamen Beziehungen sowie der Beziehungen mehrerer Personen beiden Geschlechts (sog. Kommune) beinhaltet. In dem selben Artikel verbindet der Verfassungsgeber mit der Ehe die Institution der Familie, der Mutterschaft und der Elternschaft, was für die Verfassungsaxiologie von sehr erheblicher Bedeutung ist und darauf hinweist, dass der Verfassungsgeber das traditionelle und seit langem gestaltete Verständnis der im Art. 18

7 T. Sokołowski, Opinia prawna w sprawie konstytucyjności ustawy z dnia 4 lutego 2011 r. Prawo prywatne międzynarodowe (druk senacki $n r$ 1111) [Gutachten in der Sache der Verfassungsmäßigkeit des Gesetzes vom 4. Februar 2011 - Internationales Privatrecht (Drucksache des Senats 1111)], [in:] Kancelaria Senatu. Biuro Analiz i Dokumentacji [Senatskanzlei. Das Studien- und Analysebüro], Opinie prawne o ustawie z dnia 4 lutego 2011 r. - Prawo prywatne międzynarodowe (druk senacki 1111) [Rechtliches Gutachten vom 4. Februar 2011 - Internationales Privatrecht (Drucksache des Senats 1111)], Warszawa: Kancelaria Senatu Februar 2011, S. 28. 
angewendeten Begriffe und die Anerkennung der Ehe und Familie als die Schlüsselinstitutionen unserer Zivilisation zum Ausdruck bringt. Das stellt einerseits zweifellos eine Reaktion auf die in totalitären Ordnungen (sowohl faschistischer, nationalsozialistischer als auch kommunistischer Provenienz) und im Zeitraum der Revolutionen aufgetretene Abwertung der Institution der Ehe und der Familie dar, anderseits bezieht es sich auf die in der Präambel hervorgehobenen Werte, insbesondere auf die Pflicht, die christlichen Werte als nationales Erbe zu berücksichtigen sowie auf die Pflicht sie als Werte, die das Bewusstsein der Identität und die Fortdauer des Volkes beeinflussen, zu schützen. Zu diesen Werten sind unzweifelhaft die Ehe und die Familie zu zählen. Wie von L. Garlicki zutreffend bemerkt wird, weist die Anknüpfung an christliche Werte in der Präambel auf „den Willen der Verbindung der Verfassung mit einem System von Normen und höheren (natürlichen) Werten hin, welche also außer (und über) den positiven Regelungen und Festlegungen der Auffassungs- und Anwendungsweise der Verfassungsbestimmungen stehen"8.

Die Verbindung der Institution der Ehe und Familie hat übrigens eine Akzeptanz in der ständigen Rechtsprechung polnischer Gerichte. Als Beispiel genügt es, die Auffassung des Obersten Gerichts noch aus dem Zeitraum vor dem Beschluss der geltenden Verfassung $\mathrm{zu}$ nennen: „Die Scheidungssache ist nach der Auffassung der polnischen Gesetzgebung nicht die Sache, in der es ausschließlich um die eventuelle Auflösung der Ehe geht, sondern die Sache, in der das Gericht im Zusammenhang mit dieser Entscheidung auch über das Ganze der Familiensachen für Recht erkennt (...)“9. Dagegen hat der Verfassungsgerichtshof festgelegt: „Die Rechtsinstitution der Ehe bündelt wichtige Interessen der Ehegatten, nicht nur ein persönliches

8 L. Garlicki, Besprechung der Einführung, [in:] L. Garlicki (Red.), Konstytucja Rzeczpospolitej Polskiej. Komentarz [Verfassung der Republik Polen. Kommentar], Warszawa: Wyd. Sejmowe 2005, S. 11-12.

9 Beschluss der Großen Kammer für Zivilsachen des Obersten Gerichts vom 12.10.1970, III CZP 6/70, Orzecznictwo Sądu Najwyższego - Izba Cywilna/Pracy [Zeitschrift Rechtsprechung des Obersten Gerichts - Zivil- und Arbeitskammer, OSNCP] 1971, Nr. 7-8, Pos. 117. 
Interesse, sondern auch ein Vermögensinteresse (u.a. elterliche Sorge, Trennung des Vermögens, Unterhaltspflicht von Kindern und des anderen Ehegatten ${ }^{\star \prime} 10$.

Das verfassungsrechtliche Konzept der Ehe strahlt auf andere Verfassungsnormen aus, indem es die Art ihrer Auslegung bestimmt. Das betrifft auch das oft im Zusammenhang mit den sogenannten Lebenspartnerschaften genannte Gleichheitsprinzip (Art. 32 der Verfassung). In Anbetracht der Systemauslegung und ihrer Platzierung im zweitem Kapitel ist es eben unter dem Gesichtspunkt des im Art. 18 verankerten Ordnungsprinzips auszulegen.

Dieser Auffassung entspricht die in Schlussanträgen des Obersten Gerichts enthaltene Äußerung: „Die verfassungsrechtliche Bedeutung der Ehe für das Bestehen der Familie begründet die besonderen protektionistischen und unterstützenden Regelungen im Rechtssystem, insbesondere im Bereich des Familienrechts, des Erbrechts bezüglich der Wohnungen, des Sozial- und sogar des Steuerrechts. Sie können unter Bezugnahme auf andere Verfassungsnormen, insbesondere Art. 32 der Verfassung (Gleichheitsprinzip, Verbot der Diskriminierung) nicht beanstandet werden, wie aus dem Art. 18 der Verfassung die Einräumung des Schutzes und der Erleichterungen der heterosexuellen Ehe folgt (und das ist ein Ordnungsprinzip), die den Paaren nicht zustehen, die eine Ehe nicht schließen wollen oder nicht können"11. Eine solche Methode erkennt der Europäische Gerichtshof für Menschenrechte an. Er stellt fest: "Grundsätzlich ist der Schutz der Familie im traditionellen Sinn ein gewichtiges und legitimes Ziel. Der Staat hat hierbei aber eine Vielzahl an Möglichkeiten und Maßnahmen zu setzen“12. In der Situation, in der der Europäische Gerichtshof für Menschenrechte die Notwendigkeit der Einhaltung des in der Konvention verankerten Gleichheitsprinzips wahrnimmt, entdeckt er die Grundlagen für den Schutz der tatsächlichen

\footnotetext{
10 Fußnote 3.

11 Biuro Studiów i Analiz Sądu Najwyższego [Das Oberste Gericht, Das Studienund Analysebüro], Opinia o poselskim projekcie ustawy "O umowie związu partnerskiego" [Das Gutachten über den Gesetzentwurf des Sejms "Über den Vertrag der Lebenspartnerschaft"], ein vervielfältigtes Skript, BSA I-021-135/11, S. 8.

12 Sache Kozak gegen Polen, Beschwerdesache 13102/02.
} 
Interessen von zwei zusammenlebenden Personen im Art. 8 der Konvention (Schutz des Familienlebens), indem er sich mit praktischen Problemen auseinandersetzt (,Den generellen Ausschluss von in einer homosexuellen Beziehung lebenden Personen von der Nachfolge in einem Mietverhältnis kann der Gerichtshof nicht als für den Schutz der traditionellen Familie notwendig akzeptieren“13). Hinzuzufügen ist, dass der Begriff „das Familienleben“ nach Auffassung des Gerichtshofs, im Sinne des Art. 8 der Konvention, außer den Blutsbanden oder der Ehe auch jene Situationen betrifft, in denen "eine tatsächliche Bindung das Familienleben bestimmt" ${ }^{\prime \prime}$.

Das verfassungsrechtliche Konzept der Ehe strahlt auch auf einfache Gesetze aus, indem es die Richtung der Handlung des Gesetzgebers bestimmt. Die Exemplifikation des oben Genannten kann der Art. 23 des Familienund Vormundschaftsgesetzbuches sein, indem der Inhalt der Institution der Ehe bestimmt wird, die auf dem gemeinsamen Leben, der gegenseitigen Hilfe und Treue sowie dem gemeinsamen Wirken für das Wohl der Familie beruht. Das Oberste Gericht hat festgestellt, dass unter einer ehelichen Gemeinschaft die tatsächlich die beiden Ehegatten verbindende Beziehung zu verstehen ist, die ein gemeinsames Wohnen und Haushaltsführung, eheliche Lebensgemeinschaft, eheliche Treue und Hilfe beim gemeinsamen Wirken für das Wohl der Familie umfasst. Das Oberste Gericht erkennt an, dass Art. 18 und Art. 71 der Verfassung, die die Grundsätze des besonderen Familienschutzes enthalten, die im Familien- und Vormundschaftsgesetzbuch dominante Forderung der Minimalisierung der Verbote der Eheschließung und die Beständigkeit der schon geschlossenen Ehen implizieren"15. In der Begründung einer Entscheidung hat das Oberste Gericht überdies festgestellt, dass unter einer ehelichen Gemeinschaft die tatsächlich

\footnotetext{
13 Ebenda.

14 Bescheid in der Sache X gegen die Schweiz vom 10.07.1978, DR 13, S. 252-253; polnischer Text: T. Jasudowicz, Prawa rodziny - prawa w rodzinie [Die Rechte der Familie und die Rechte in der Familie], Torun: TNOiK 1999, S. 30.

15 Beschluss des Obersten Gerichts vom 9.05.2002, III CZP 7/02, Orzecznictwo Sądu Najwyższego - Izba Cywilna [Zeitschrift Rechtsprechung des Obersten Gerichts Zivilkammer; OSNC] 2003, Nr. 1, Pos. 1.
} 
die beiden Ehegatten verbindende Beziehung $\mathrm{zu}$ verstehen ist, die ein gemeinsames Wohnen und Haushaltsführung, eheliche Lebensgemeinschaft, eheliche Treue und Hilfe beim gemeinsamen Wirken für das Wohl der Familie umfasst" 16 .

Die Stellung der Ehe und der Familie unter den Schutz und die Obhut der Republik Polen erlegt der öffentlichen Gewalt bestimmte Pflichten auf, die mit der Aufführung der durch den Verfassungsgeber angeführten Tätigkeitsziele dieser Gewalt verbunden sind, aber keine Grundlage dafür darstellen, um aus dem Art. 18 die subjektiven Rechte abzuleiten. In der Rechtsprechung des Verfassungsgerichthofes nimmt man an, dass Art. 18 der Verfassung grundsätzlich keine Rechte oder Freiheiten bestimmt, die den Bürgern direkt zustehen. Die in der Form der Politikprinzipien des Staates und nicht als Rechte des Einzelnen gefasste Vorschrift kann somit grundsätzlich nicht allein die Grundlage für die individuelle Geltendmachung von Ansprüchen im Wege der Verfassungsbeschwerde darstellen ${ }^{17}$. Der Empfänger des Art. 18 ist der Gesetzgeber und an ihn sind die in ihm bestimmten Ziele gerichtet. „Das Ziel der verfassungsrechtlichen Regulierung, die sich auf den Familienstatus bezieht, ist (...) die Verpflichtung für den Staat, insbesondere für den Gesetzgeber, festzulegen, solche Maßnahmen vorzunehmen, die die Bindung zwischen den die Familie gründenden Personen, insbesondere die zwischen Eltern und Kindern und zwischen den Ehegatten verstärken. (Urteil vom 18. Mai 2005, K 16/04, Orzecznictwo Trybunału Konstytucyjnego; Zbiór Urzędowy [Rechtsprechung des Verfassungsgerichtshofs - Amtliche Sammlung, OTK] Nr. 5/A/2005, Pos. 51, Pkt. III. 4), (...) Dazu können jedoch, wenigstens mittelbar durch Abschwächung der Beständigkeit von Familienbeziehungen, jene Lösungen, die die Erziehung der Kinder nur durch einen Elternteil oder auch durch beide Eltern, aber ohne Eheschließung, bevorzugen würden,

16 Urteil des Obersten Gerichts vom 6.03.1997, II UKN 17/97, Orzecznictwo Sądu Najwyższego - Izba Pracy i Ubezpieczeń Społecznych [Zeitschrift Rechtsprechung des Obersten Gerichts - Arbeits- und Versicherungskammer; OSNP] 1997, Nr. 23, Pos. 477.

17 Urteil des Verfassungsgerichtshofes vom 10.07.2000, SK 21/99, Orzecznictwo Trybunału Konstytucyjnego; Zbiór Urzędowy [Rechtsprechung des Verfassungsgerichtshof; Amtliche Sammlung, OTK] 2000, Nr. 5, Pos. 144. 
nicht führen“18. Dieser Stellungnahme entspricht die folgende Auffassung des Obersten Gerichts: [Der Grundsatz] des Eheschutzes, der im Art. 18 der Verfassung bestimmt ist, bedeutet, dass die rechtlich legalisierte Beziehung einer Frau und eines Mannes unter dem Schutz und der Obhut der Republik Polen stehen. Der Eheschutz äußert sich unter anderem darin, dass die sich aus der Ehe ergebenden rechtlichen Konsequenzen in Bezug auf die anderen Relationen keine Anwendung finden sowie dass jede Auslegung oder die Anwendung des Rechts, die zur Gleichstellung anderer Formen des gemeinsamen Wohnens mit der Situation der Ehe führen würden, unzulässig sind. Unter Berücksichtigung des Verfassungsgrundsatzes des Eheschutzes sowie der Tatsache, dass das Fehlen der rechtlichen Regelungen für nichteheliche Lebensgemeinschaften nicht als eine Rechtslücke anerkannt werden kann, ist es nicht zulässig, die Vorschriften des Eherechts (einschließlich des Ehevermögens und seiner Teilung) - auch nicht durch Analogie - auf die Beziehungen, die sich auf das Bestehen von persönlichen und wirtschaftlichen Bindungen stützen, die aber nicht Ehen sind, anzuwenden" 19 .

Die vom Verfassungsgeber angewandten zwei Begriffe - Schutz und Obhut - verweisen einerseits auf die Absicht, zwei verschiedene Aufgabenkomplexe und die ihnen entsprechenden Aktivitätssphären der Staatsorgane $\mathrm{zu}$ bestimmen, anderseits ordnet die Konjunktion zwischen den beiden Wörtern diese Organe an, verschiedene Formen ihrer Tätigkeiten zu verbinden. In Anbetracht des oben Genannten ergibt es keinen Sinn für die praktische Rechtssetzung und die Anwendung des Rechts, die beiden Ziele der öffentlichen Gewalt durch die Verdeutlichung der Unterschiede zwischen beiden in der Verfassung angewandten Begriffen abzutrennen. Im Übrigen ist diese Tendenz auch in der gerichtlichen Rechtsprechung und beim Verfassungsgerichthof sichtbar. Generell gilt, dass sich aus dem Art. 18 der Verfassung

18 Entscheidung des Verfassungsgerichtshofes vom 12.04.2011, SK 62/08, Orzecznictwo Trybunału Konstytucyjnego; Zbiór Urzędowy, Seria A [Rechtsprechung des Verfassungsgerichtshof; Amtliche Sammlung, Serie A, OTK-A] 2011, Nr. 3, Pos. 22.

19 Urteil des Obersten Gerichts vom 6.12.2007, IV CSK 301/07, Orzecznictwo Sądu Najwyższego - Izba Cywilna [Zeitschrift Rechtsprechung des Obersten Gerichts - Zivilkammer; OSNC] 2009, Nr. 2, Pos. 29. 
die der öffentlichen Gewalt auferlegten Sicherungspflichten der Ehe und der Familie vor allen Gefahren für ihr normales Funktionieren ergeben. Diese Pflichten umfassen nicht nur die Abwehr vor Gefahren seitens fremder Subjekte gegenüber der Familie (Moralgefahr für Kinder), sondern auch das Tätigwerden der staatlichen Organe in der sozialen und wirtschaftlichen Sphäre, die Durchsetzung der das Funktionieren der Ehe und der Familie unterstützenden Sozial- und Wirtschaftspolitik sowie die Stärkung der Bindungen zwischen Ehegatten und Familie. Diese Maßnahmen beruhen im Lichte der Rechtsprechung der Verfassungsgerichtshofes u.a. auf der Durchsetzung einer entsprechenden Steuerpolitik zugunsten der Ehen und Familien, der Rechtsetzung, um den Wohnbedarf der Ehen und Familien zu erfüllen, der Gewährung der Sozialleistungen, die die wirtschaftliche Lage der Familien verbessern, dem Schutz der Ehegattenrechte in ihren Verhältnissen zueinander. Es ist noch dabei $\mathrm{zu}$ betonen, dass sich die Pflichten der öffentlichen Gewalt aus dem Art. 18 ergeben und auf jede Ehe und jede Familie auf dem Gebiet Polens beziehen. Das betrifft auch die Familien von Ausländern.

Im Zusammenhang damit, dass im Art. 18 der Verfassung die Ehe und Familie vom Staat unter Schutz und Obhut gestellt wurden, nimmt das Verhältnis der Republik Polen $\mathrm{zu}$ den tatsächlich bestehenden Emotional- und Vermögensbeziehungen von zwei Personen, die eine nichteheliche Form der Lebensgemeinschaft darstellen, eine besondere Bedeutung an. In der Rechtslehre wird betont, dass sich aus dem Art. 18 weder ein Verbot des Bestehens solcher Beziehungen²0, noch ein Gebot ihrer Bekämpfung folgern lässt. Mit dieser Lehrmeinung steht die gerichtliche Rechtsprechung im Einklang. Es ist in diesem Zusammenhang festzustellen, dass der verfassungsrechtliche Schutz der Ehe nicht bedeutet, dass andere Formen der gemeinsamen Lebensgemeinschaft als die Ehe durch das Recht verboten sind“21.

In der gerichtlichen Rechtsprechung, welche sich auf die Verfassungsaxiologie bezieht, werden sie unterschiedlich zur Ehe

20 Vgl. P. Winczorek, Komentarz do Konstytucji Rzeczypospolitej Polskiej $z$ dnia 2 kwietnia 1997 roku [Kommentar zur Verfassung der Republik Polen vom 2. April 1997], Warszawa: Liber 2008, S. 54.

21 Fußnote 19. 
behandelt. „Das polnische Recht enthält weder eine umfassende noch fragmentarische Regelung der nichtehelichen Personenund Vermögensgemeinschaften und deshalb werden sie als tatsächliche Beziehungen rechtlich indifferent behandelt. Mit Rücksicht darauf, dass das Recht ihren Status nicht regelt und dass es unzulässig ist, auf sie die Vorschriften über die sich aus der Eheschließung ergebenden Vermögensverhältnisse anzuwenden, ist es notwendig, die Berechnungsgrundlagen im Bereich des Zivilrechts $\mathrm{zu}$ suchen. (...) Falls keine rechtlichen Regelungen der nichtehelichen Personenund Vermögensgemeinschaften bestehen, wurden die gewissen Arten der Erfassung und der Behandlung einer solchen Lebensgemeinschaft in der Rechtsprechung und in Lehrmeinungen herausgearbeitet, die als freie Lebensgemeinschaft [polnisch: konkubinat] bezeichnet wird. $\mathrm{Zu}$ den Kriterien der freien Lebensgemeinschaft werden grundsätzlich keine formellen Grundlagen der Lebensgemeinschaft von Partnern sowie keine Beschränkungen bezüglich der Beendigung der Beziehung, ihrer Stabilität, des Bestehens der Personen- und Vermögensgemeinschaften und des Unterschiedes des Geschlechts der Partner gezählt. Der in den Lehrmeinungen und in der Rechtsprechung herausgearbeitete Begriff der freien Lebensgemeinschaft betrachtet den Unterschied des Geschlechts von Lebensgefährten als ihr wesentliches Merkmal“22.

Wie von L. Garlicki zutreffend vorgetragen, stellt der Art. 18 der Verfassung das Element der Verfassungsaxiologie dar. Er enthält in sich einen konkreten Regelungsgehalt und verbietet es, den tatsächlichen Beziehungen der Personen verschiedenen Geschlechts (freie Lebensgemeinschaft) oder gleichen Geschlechts den Charakter einer Ehe $\mathrm{zu}$ verleihen ${ }^{23}$.

Art. 18 der Verfassung, indem er den öffentlichen Gewaltorganen der Republik Polen auferlegt, Ehe und Familie unter Schutz und Obhut zu stellen, impliziert das Verbot, durch diese Organe Maßnahmen zu ergreifen, die das Ziel haben, für die tatsächlich bestehenden Emotionalund Vermögensbeziehung von zwei Personen, die eine nichteheliche Form der Lebensgemeinschaft darstellt, solche Regelungen zu schaffen,

\footnotetext{
22 Ebenda.

23 Garlicki (Red.), Fußnote 8, Art. 2, S. 2-3.
} 
die sie in Rechtsgestalt an die Ehe bezüglich der Bestimmung von Rechten und Pflichten der Personen, die sich in dieser Beziehung befinden sowie der Frage ihrer Schließung oder Auflösung, anpassen oder sie dieser gleichsetzen.

Die abgelehnten Gesetzentwürfe über Lebenspartnerschaften und die Einführungsgesetze $\mathrm{zu}$ diesen Gesetzen sind jedoch in diese Richtung gegangen. Es genügt hier die folgende Regulierung anzugeben:

- die Schließung einer Lebenspartnerschaft auf dem Wege der vor dem Leiter des Standesamtes abgegebenen Willenserklärung (der Entwurf der Bürgerplattform [Platforma Obywatelska] hat einen alternativen Vertragsabschluss vor dem Notar vorgesehen, aber der Vertrag soll in das vom Leiter des Standesamtes geführte Register eingetragen werden),

- Personen, die eine Lebenspartnerschaft schließen wollen, sollen dem Leiter des Standesamtes die zur Beziehungsschließung notwendigen Urkunden vorlegen, von denen im Gesetz - das Recht über Personenstandsbücher - die Rede ist (Art. 3 Abs. 3 des Gesetzentwurfs der Palikot-Bewegung [Ruch Palikota]). Der Entwurf der Bürgerplattform sah im Art. 6 Abs. 1 direkt vor: „Personen, die einen Vertrag der Lebenspartnerschaft abschließen wollen, sollen dem Leiter des Standesamtes die zum Vertragsabschluss der Lebenspartnerschaft notwendigen Urkunden zum Abschluss dieses Vertrages vorlegen oder vorzeigen, wie die Personen, die eine Ehe schließen wollen",

- Der Hinderniskatalog für Personen, die eine Lebenspartnerschaft schließen wollen, war dem Hinderniskatalog für Personen, die eine Ehe schließen wollen, ähnlich,

- Die Rechte und Pflichten der Personen, die eine Lebensgemeinschaft geschlossen haben, waren mit Rechten und Pflichten der Ehegatten vereinbar,

- Die Auflösung der Lebenspartnerschaft sollte u.a. entweder im Wege der abgegebenen übereinstimmenden Erklärungen der Personen, die sie geschlossen haben (vor dem Notar, vor dem Leiter des Standesamtes) oder bei mangelnder Einigung sollte die Auflösung durch das Gericht erfolgen. Die letzte Variante ist der Auflösung der Ehe ähnlich. 
Außerdem ist darauf hinzuweisen, dass die Einführung der Lebenspartnerschaften nach der Intention der Verfasser die Notwendigkeit der wesentlichen Novellierung von zahlreichen Gesetzen verursachen sollte, indem sie die unter dem Einfluss der oben erklärten Verfassungsaxiologie entstandenen Regelungen enthalten. Die Menge der novellierten Gesetze schwankte zwischen 50 (Entwurf der Bürgerplattform) und 70 (Entwurf der Palikot-Bewegung) bis 154 (Entwurf der Sozialdemokratischen Partei [Sojusz Lewicy Demokratycznej]). Unter diesen Umständen ist es natürlich schwer über die Ausstrahlung des Verfassungsprinzips $\mathrm{zu}$ sprechen, indem es die Pflicht des Staates zum Schutz und zur Obhut gegenüber der Ehe und Familie einführt, für die es die Grundlage darstellt.

Aus vorstehenden Ausführungen ergibt sich eindeutig, dass die Annahme eines die Institutionalisierung der tatsächlich bestehenden Personen- und Vermögensgemeinschaften von zwei Personen vorsehenden Gesetzes, die im Art. 18 der Verfassung der Republik Polen ausgedrückte Pflicht des Staates zum Schutz und Obhut gegenüber der Ehe und Familie verletzen würde. Es geht hier nicht um die Verletzung der Eherechte, sondern um die Ausdehnung der rechtlichen Regelungen über die Ehegatten auf andere Formen der Lebensgemeinschaften, was die Idee des Schutzes und der Obhut der Ehe gerade durch diese Regulierungen widerlegt. Der Eheschutz äußert sich darin, dass die Organe des Gesetzgebers keine Gesetze umfassend annehmen können, indem sie andere Formen des gemeinsamen Wohnens als die Ehe regeln sowie durch ihre Institutionalisierung $\mathrm{zu}$ ihrer Gleichstellung mit der Ehe oder mindestens $\mathrm{zu}$ ihrer erheblichen Angleichung an die Ehe führen. Das gilt insbesondere für die Situation, in der die Institutionalisierung der anderen Formen der gemeinsamen Lebensgemeinschaft als der Ehe auf der Schließung einer Lebenspartnerschaft vor dem Leiter des Standesamtes und ihrer Auflösung durch das Gericht und der Einräumung der Mehrheit von Rechten und Pflichten der Ehegatten für die Parteien dieser Beziehung beruhen würde. Mit dem Art. 18 der Verfassung verbindet sich noch ein maßgebliches Kriterium der nach der Auffassung des Obersten Gerichts wahrgenommenen Gesetzgebertätigkeit: „Aus dem Art. 18 der Verfassung 
ergibt sich eine Richtlinie für den Gesetzgeber, damit das geltende Recht zur Eheschließung und zur 'Legalisierung' der tatsächlichen Beziehungen durch die Eheschließung 'ermutigt' wird. (...) Die Institutionalisierung der heterosexuellen freien Lebensgemeinschaft ist im Zusammenhang mit dem Inhalt des Art. 18 der Verfassung nicht zulässig. Indirekt würde durch die Schaffung einer Alternative für die Ehe (die auf fast allen sich aus ihr ergebenden Vorteilen bei keinen vergleichbaren Pflichten und Hindernissen im Fall der Auflösung der Beziehung beruht) - die Verringerung ihrer 'Attraktivität' erfolgen"24. Im Lichte der genannten Anforderungen bezüglich der Lebenspartnerschaften ist es schwer, die rationale Begründung der Abneigung der Personen, die solche Beziehung schließen wollen, gegen die Institution der Ehe wahrzunehmen, wenn sie für die Schließung der Lebenspartnerschaft die gleichen Dokumente wie die Heiratswilligen vorlegen sollen. Sie sollten die Lebenspartnerschaft im Standesamt schließen, die den Ehegatten ähnlichen Rechte und Pflichten erlangen und sie könnten ihre Partnerschaft vor Gericht auflösen. Die Institutionalisierung der Lebenspartnerschaften würde in dieser Situation eine Verletzung der dem Gesetzgeber auferlegten Richtlinie darstellen, von der das Oberste Gericht spricht.

Aus dem Art. 18 der Verfassung ergibt sich, dass die Organe, die das Recht anwenden, die Auslegung der bestehenden Rechtsnormen nicht anwenden können, wenn durch verschiedene Formen des gemeinsamen Wohnens in rechtlichen Verhältnissen die gleiche oder eine ähnliche Stellung wie die der Ehe erzielt würde. Das gilt insbesondere für die Nichtanwendung des Familien- und Vormundschaftsgesetzbuches auf die nichtehelichen Personen- und Vermögensgemeinschaften. Auf die Streitbeilegung zwischen den solche Beziehung bildenden Personen sind die Vorschriften der dem Inhalt nach den in dieser Beziehung gestalteten Verhältnissen entsprechenden Gesetze anzuwenden ${ }^{25}$. In Lehrmeinungen wird zutreffend betont: „Der Versuch

\footnotetext{
24 Biuro Studiów i Analiz Sądu Najwyższego [Das Oberste Gericht, Das Studienund Analysebüro], Opinia o poselskich projektach ustawy "O zwiazkach partnerskich" oraz "Przepisy wprowadzające ustawe o związach partnerskich" [Das Gutachten über Gesetzentwürfe des Sejms "Über die Lebenspartnerschaften” und "Die Einführungsvorschriften zum Gesetz über Lebenspartnerschaften“], ein vervielfältigtes Skript, BSA I-021-133-124/12, S. 11-14. 25 Siehe z.B. Fußnote 19.
} 
der begrenzten Auslegung des Art. 18, indem sie zur Darstellung - 'neben' der Ehe - eines Raumes für andere institutionelle Beziehungen als der Ehe führt, führt (...) direkt dazu, die fundamentalen Ordnungsprinzipien, die den ausschließlichen Charakter der Ehe bestimmen, in Frage zu stellen. Der Umstand, dass in manchen Ländern in diesem Bereich andere Ordnungsprinzipien angenommen und neue terminologische Konventionen eingeführt wurden, stellt hier kein Argument zugunsten der abweichenden Auslegung des Art. 18 dar, dessen Inhalt und Funktion ganz klar ist" 26 .

Das schließt jedoch nicht aus, nach der Rechtsprechung des EGMR, die detaillierten Probleme der in nichtehelichen Emotionalund Vermögensbeziehungen lebenden Personen zu lösen. Jedes Mal ist dabei darauf $\mathrm{zu}$ achten, solchen Beziehungen die Berechtigungen zu gewähren, die einen Bestandteil des Wesens der Ehe und des durch die Nebenregulierung des im Art. 18 bestehenden und gefestigten Wertschutzsystems darstellen, das mit Ehe und Familie verbunden ist. Das Oberste Gericht hat in seinen Schlussanträgen ausgeführt: „Für manche nicht direkt mit dem besonderen Eheschutz verbundenen Änderungsverschläge bestehen aufgrund des Näheverhältnisses zwischen den in einer Lebensgemeinschaft lebenden Personen oder aus einem anderen sozialen Grund keine Zweifel“ 27 .

Die Gewährung der durch das Gesetz den in heterosexuellen Lebenspartnerschaften lebenden Personen zuerkannten Berechtigungen verletzt jedoch den die Ehe hervorhebenden Art. 18 der Verfassung, wenn sie zur Institutionalisierung dieser Beziehungen führt und diese Beziehungen an die Ehe anpasst. Die Gewährung der konkreten Berechtigungen (z.B. bezüglich des Vermögenscharakters) kann für verfassungsmäßig anerkannt werden, wenn sie nicht gegen das verfassungsrechtliche Konzept (ein Verfassungsmodell) der Ehe und Familie verstößt.

Weder Art. 18 der Verfassung noch irgend eine andere Norm definiert den Begriff der Familie. In der Rechtslehre

26 Sokołowski, Fußnote 7, S. 31.

27 Biuro Studiów i Analiz Sądu Najwyższego [Das Oberste Gericht, Das Studienund Analysebüro], Fußnote 11, S. 26. 
fehlt es an einer allgemein angenommenen Definition der Familie. Aus dem Art. 18 wird meistens abgeleitet, dass die Familie die Ehegatten sind und wenn sie Kinder haben - dann sie und die Kinder. Darauf verweist die Reihe der Schutzgüter im Art. 18 - Ehe, Familie, Mutterschaft, Elternschaft. Das impliziert die Anerkennung der folgenden Auffassung: „Verfassungsmäßig ist die Gründung der Familie durch die in der typischen Situation die Fortpflanzung-, Sozialisationsund Fürsorgefunktion realisierende Ehe“ 28 .

Die Verfassung erkennt die Familie als das grundlegende Element der Gesellschaftsstruktur an und betont ihre Rolle bei der Kindererziehung ${ }^{29}$. Somit kann man zu dem Begriff der Familie eine Frau und einen Mann zählen, die zwar gemeinsam leben, aber die nicht Ehegatten sind (freie Lebensgemeinschaft). Sie werden jedoch zur Familie, wenn sie Kinder haben werden. Diese Familie „erstreckt sich (...) dann auf die Mutterschaft der Lebensgefährtin und auf die anerkannte (...) Vaterschaft des Lebensgefährten“30.

Nebenbei ist daran zu erinnern, dass sowohl die völkerrechtlichen Universalrechtsakte als auch die EMRK dem traditionellen Familienkonzept Ausdruck verliehen haben. Art. 9 der Charta der Grundrechte bestimmt, dass das Recht, eine Ehe einzugehen, und das Recht, eine Familie zu gründen, nach den einzelstaatlichen Gesetzen gewährleistet werden, welche die Ausübung dieser Rechte regeln. Somit kann das EU-Recht in die durch das polnische Familienrecht bestimmte Materie nicht eingreifen. Dazu ist zu ergänzen, dass Polen und Irland das sogenannte britische Protokoll unterzeichnet haben, das die Anwendung einiger Bestimmungen der Charta der Grundrechte auf dem Gebiet dieser drei Staaten bezüglich

28 Biuro Studiów i Analiz Sądu Najwyższego [Das Oberste Gericht, Das Studienund Analysebüro], Opinia o poselskim projekcie ustawy "O umowie związk partnerskiego" (wersja projektu z 22 maja 2012 r.) [Das Gutachten über den Gesetzentwurf des Sejms „Über den Vertrag der Lebenspartnerschaft" (die Version der Entwürfe vom 22. Mai 2012)], ein vervielfältigtes Skript, BSA I-021-227-228/12, S. 3.

$29 \mathrm{Vgl}$. T. Smyczyński, Rodzina i prawo rodzinne $w$ świetle nowej Konstytucji [Familie und Familienrecht im Lichte der neuen Verfassung], Państwo i Prawo [Staat und Recht] 1997, H. 11-12, S. 185.

30 Siehe A. Grzejdziak, Prawo do wychowania w rodzinie [Das Recht auf Erziehung in der Familie], [in:] B. Banaszak, A. Preisner (Red.), Prawa i wolności obywatelskie w Konstytucji RP [Bürgerliche Rechte und Freiheiten in der Verfassung der Republik Polen], Warszawa: C.H. Beck 2002, S. 464. 
der Sozialrechte, des Umwelt- und Verbraucherschutzes ausschließt. Das Protokoll verbietet auch, die im Inland geltenden rechtlichen Vorschriften auf der Grundlage der Charta durch jedes andere Gericht, darunter auch durch den Gerichtshof der Europäischen Union, für ungültig zu erklären. Polen hat darüber hinaus eine einseitige Erklärung abgegeben, dass die Charta in keiner Weise das Recht der Mitgliedstaaten, in den Bereichen der öffentlichen Sittlichkeit, des Familienrechts sowie des Schutzes der Menschenwürde und der Achtung der körperlichen und moralischen Unversehrtheit Recht zu setzen, berührt.

Nach der gegenwärtig geltenden Rechtslage liegt keine legale Definition des Begriffs der Familie vor.

Aus dem Art. 23 des Familien- und Vormundschaftsgesetzbuches ergibt sich, dass die Ehegatten die Familie gründen und somit sie und ihre Kinder, wenn sie welche haben, die Familie darstellen. Wieder gibt der Gesetzgeber für die Bedürfnisse der einzelnen Rechtszweige noch andere Definitionen vor, je nach den Zielen, denen die Gesetze dienen sollen. So kann beispielsweise auf den Art. 3 Pkt. 16 des Gesetzes vom 28. November 2003 über Familienleistungen hingewiesen werden, indem er vorsieht, dass unter einer Familie folgende Familienmitglieder zu verstehen sind: Ehegatten, Eltern der Kinder, die tatsächliche Pflegeperson des Kindes oder andere unterhaltsberechtigte Kinder im Alter bis zur Vollendung des 25. Lebensjahres sowie jenes Kind, welches das 25. Lebensjahr vollendet hat und über einen ärztlichen Befund über einen schweren Behinderungsgrad verfügt, wenn der Familie im Zusammenhang mit dieser Behinderung eine Pflegeleistung zusteht.

Eingedenk der Unterschiede in legalen Definitionen resümiert das Oberste Gericht: „Man kann annehmen, dass nach Rechtsakten in der großen Mehrzahl zur Familie gehören: Ehegatten, ihre gemeinsamen Kinder, Kinder eines Eheteiles, adoptierte Kinder, die im Rahmen der Pflegefamilie zur Erziehung angenommenen Kinder, die unter der (rechtlichen) Obhut stehenden Kinder und manchmal sogar die zur Erziehung und zum Unterhalt angenommenen fremden Kinder, wenn die Eltern nicht mehr leben oder ihren Kinder keinen Unterhalt gewähren können oder auch ihnen das elterliche Sorgerecht entzogen 
oder beschränkt wurde“31. Das Oberste Gericht knüpf hier deutlich an die Rechtsprechungslinie an, die den Begriff die Familie mit der Ehe verbindet. In der so begriffenen Familie - im Falle der Ehegatten, welche Kinder haben - kann das Wohl der Kinder dann für die Fortführung der Ehe sprechen und die Verweigerung ihrer Auflösung begründen. In diesem Zusammenhang ist auf die ständige Auffassung des Obersten Gerichts und auf seine Rechtsprechung mit Richtliniencharakter hinzuweisen, die die gerichtliche Rechtsprechung zur Verstärkung des Familienschutzes ausrichten sollen, insbesondere durch folgende Fragestellungen: Bewirkt die Scheidung die Abschwächung der Beziehung zu den Kindern von Seiten desjenigen Ehegatten, bei dem die Kinder nicht verbleiben; werden nach der Scheidung die wirtschaftlichen und emotionalen Bedürfnisse von Kindern mindestens in diesem Bereich wie gegenwärtig gedeckt; wird für die Kinder die Aufrechterhaltung des bisherigen Standes günstiger sein als die Entscheidung für eine Scheidung 32 ?

In der oben genannten Begründung der Entscheidung hat das Oberste Gericht die mit der Ehe verbundene Auffassung jedoch erweitert, indem es feststellte: „Die Entwicklung der freien Lebensgemeinschaft, die eine dauerhafte Stelle in einem Bewertungs- und Moralnormensystem der gegenwärtigen Gesellschaft gefunden hat, hat ihre Quelle in der Änderung des Politik- und Wirtschaftssystems sowie in den kulturellen Änderungen, insbesondere des Bewusstseins der Gesellschaft. In der gegenwärtig geltenden Rechtslage kann man bei der Formulierung des Begriffs 'Familie' - worauf in Lehrmeinungen hingedeutet wird - folgende Kriterien anwenden: Verwandtschaft, Ehe, Adoption, Verschwägerung, Pflegefamilie und das Zusammenleben in einem gemeinsamen Haushalt. Somit

\footnotetext{
31 Urteil des Obersten Gerichts vom 13.04.2005, IV CK 648/04, Orzecznictwo Sądu Najwyższego - Izba Cywilna [Zeitschrift Rechtsprechung des Obersten Gerichts Zivilkammer; OSNC] 2006, Nr. 3, Pos. 54.

32 Siehe Entscheidungen des Obersten Gerichts: vom 18.03.1968, III CZP 70/66, Orzecznictwo Sądu Najwyższego - Izba Cywilna/Pracy [Zeitschrift Rechtsprechung des Obersten Gerichts - Zivil- und Arbeitskammer, OSNCP] 1968, Nr. 6, Pos. 77; vom 9.06.1976, III CZP 46/75, Orzecznictwo Sądu Najwyższego - Izba Cywilna/Pracy [Zeitschrift Rechtsprechung des Obersten Gerichts - Zivil- und Arbeitskammer, OSNCP] 1976, Nr. 9, Pos. 184.
} 
kann man die Definition der Familie als die kleinste Gesellschaftsgruppe, die mit der persönlichen und wirtschaftlichen Gemeinschaft und dem nicht nur verwandtschaftlichen Nähegefühl zusammenhängt, akzeptieren“33. Das Oberste Gericht hat hier zweifellos auf seine ständige Rechtsprechung verwiesen, nach der die Verwandtschaft kein grundlegendes oder einziges Kriterium der Nähe und der Zugehörigkeit zur Familie darstellt ${ }^{34}$. In diese Richtung geht auch der Verfassungsgerichtshof, indem er feststellt, dass die Familie, im Lichte der verfassungsmäßigen Vorschriften, ,jede ständige Beziehung von zwei oder mehreren Personen darstellt, die mindestens aus einer erwachsenen Person und dem Kind besteht und die auf emotionalen, rechtlichen Bindungen und überwiegend auch auf Blutsbanden basiert. Die Familie kann eine 'vollständige' Familie darunter auch eine 'Mehrkinderfamilie' oder eine 'unvollständige' Familie sein. Die 'vollständige' Familie besteht aus zwei Personen, die in einem gemeinsamen Haushalt leben, die mit Gefühlsbindungen und mit einem von ihnen gemeinsam $\mathrm{zu}$ erziehenden Kind (Kindern) verbunden sind. Hingegen bildet die 'unvollständige' Familie eine erwachsene Person und ein von ihr zu erziehendes Kind (Kinder)" 35 .

Für die Erweiterung des Begriffs der Familie spricht auch die Hervorhebung der Bedeutung der Elternschaft in Art. 18 der Verfassung. Der verfassungsmäßige Schutz bedeutet, dass mit ihm sowohl die Mutterschaft und die Vaterschaft, die Rechte der Mutter und des Vaters als auch der Schutz der Kinderrechte erfasst wird. Unter Berücksichtigung des oben Genannten hat das Verfassungsgericht zu Recht ausgeführt: „Nach der allgemein übernommenen Auffassung kann die umfassende Realisierung des Wohles des Kindes durch die Gewährleistung der Möglichkeit seiner Erziehung in der Familie, vor allem in einer natürlichen Familie ausgeübt werden, also durch das elterliche Sorgerecht, das von den mit dem Kind in biologischer Bindung stehenden Personen ausgeübt wird. Die Analyse der Filiationsmechanismen, auf die sich die traditionellen Familienverhältnisse

\footnotetext{
33 Fußnote 31.

34 Siehe z.B. Urteil des Obersten Gerichts vom 10.12.1969, III PRN 77/69, Orzecznictwo Sądu Najwyższego - Izba Cywilna/Pracy [Zeitschrift Rechtsprechung des Obersten Gerichts Zivil- und Arbeitskammer, OSNCP] 1970, Nr. 9, Pos. 60.

35 Fußnote 18.
} 
stützen, drückt recht offensichtlich die Tendenz dazu aus, damit die rechtlichen Relationen auf der Linie ein Elternteil und ein Kind der biologischen Wirklichkeit entsprechen. Das bedeutet nicht, dass der Primat von der natürlichen Familie und der biologischen Bindung als der Gestaltungsgrundlage der Familienverhältnisse einen absoluten Charakter hat, der immer und unter allen Umständen den Wert der Familienrelationen überwiegen muss, die in Anlehnung an andere Kriterien, die auf den Schutz der Werte, wie der Nähe und der Stabilität der Familienverhältnisse, der Sicherheit des Kindes, der angemessenen Erziehungs- und Entwicklungsbedingungen etc. verweisen, gestaltet werden"36. Aus dieser Aussage kann gefolgert werden, dass in der im Art. 18 der Verfassung enthaltenen Formulierung, die die Elternschaft hervorhebt, also die die Eltern und Kinder verbindenden Beziehungen, nicht die Ausschließung der Möglichkeit des erweiterten Verstehens des Begriffs der Familie in Gesetzen durch den Verfassungsgeber gesehen werden kann, der die auf Grundlage der Blutsbande entstehenden Generationenbeziehungen sowie die Relationen aus dem Adoptionsverhältnis berücksichtigt. Die Elternschaft entscheidet über das Bestehen der Familie, wenn die Verwandtschaftsbindung das Kind nur mit einem Elternteil verbindet. Das hat der Verfassungsgerichtshof in der oben genannten Entscheidung (unvollständige Familie) hervorgehoben und die ordentlichen Gerichte vertreten auch einen ähnlichen Standpunkt. Als Beispiel nenne ich den folgenden Begründungsausschnitt einer Entscheidung: „Der Gesetzgeber hat anerkannt, dass in der Situation, wenn die das elterliche Sorgerecht allein ausübenden Vater oder Mutter erziehungsfähig und lebenstüchtig sind, verletzen sie nicht das Interesse des Kindes und schützen ausreichend sein Gut"37. Das bedeutet, dass sowohl die biologischen als auch rechtlichen Bindungen der Verwandtschaftsverhältnisse, die im Wege der Adoption festgestellt wurden, über das Bestehen der Familie entscheiden, selbst wenn zwischen

36 Urteil des Verfassungsgerichthofes vom 28.04.2003, K 18/02, Orzecznictwo Trybunału Konstytucyjnego; Zbiór Urzędowy, Seria A [Rechtsprechung des Verfassungsgerichtshofs, Amtliche Sammlung, Serie A, OTK-A] 2003, Nr. 4, Pos. 32.

37 Urteil des Bezirksgerichts vom 18.01.2011, I C 1121/08, Online-Version: www.bialystok.so.gov.pl [Zugang am 14.03.2014]. 
den Eltern kein Eheverhältnis vorliegt. Die (biologischen und rechtlichen) Verwandtschaftsverhältnisse entscheiden auch über das Vorliegen einer Familie, selbst wenn sie aus einem Elternteil und einem Kind oder Kindern besteht.

Dieser Gedanke bedeutet jedoch nicht, dass für das Bestehen der Familie die Elternschaft notwendig ist, das heißt das Vorhandensein von Kindern. Wichtig sind auch, wovon in der Antwort auf die Frage 1 die Rede war, die aus der gemeinsamen ehelichen Lebensgemeinschaft entstandenen Verhältnisse. An dieser Stelle ist der Beschluss der Großen Kammer für Zivil- und Verwaltungssachen des Obersten Gerichts von Bedeutung 38 , in dem das Oberste Gericht der Auffassung ist, dass von der Familie grundsätzlich dann gesprochen werden kann, wenn die Familie mit einer gemeinsamen Lebensgemeinschaft verbunden ist. Nämlich nur in dieser Situation entstehen die Bedürfnisse bezüglich der Gruppe der die Familie bildenden Personen. Dabei hat es keine Bedeutung, dass die Ehe kinderlos ist oder zwar Kinder hat, aber diese Kinder sich alleine unterhalten (arbeiten, erwerbstätig sind). Die kinderlosen Ehegatten stellen eine Familie dar.

Im Lichte der vorstehenden normativen Regelungen, der Meinungen der Rechtslehre und der gerichtlichen Rechtsprechung kann festgestellt werden, dass die Familie im Sinne des Art. 18 der Verfassung nicht nur die auf die Ehe gestützte Familie bedeutet, sondern auch die Beziehung einer Frau und eines Mannes, die nicht die Form der Ehe hat, wenn sie mit einem gemeinsamen Kind (Kinder) - Elternschaft verbunden ist. Die Familie ist auch eine kinderlose Ehe und auch die Ehe und die gemeinsamen Kinder der Ehegatten sowie die Kinder eines der beiden Elternteile. Hingegen stellen die nicht ehelichen Partnerschaften einer Frau und eines Mannes, in denen es keine Kinder gibt, keine Familie dar.

Die Verfassung enthält zwar keine Bestimmungen bezüglich der Lebenspartnerschaften, aber aus vorstehenden Erwägungen ergibt sich, dass nach der gerichtlichen Rechtsprechung und nach

38 Vom 16.12.1987, III CZP 91/86, Orzecznictwo Sądu Najwyższego - Izba Cywilna/Pracy [Zeitschrift Rechtsprechung des Obersten Gerichts - Zivil- und Arbeitskammer, OSNCP] 1988, Nr. 4, Pos. 42. 
überwiegender Auffassung in der Rechtslehre, der zufolge Art. 18 der Verfassung die Institutionalisierung der heterosexuellen Personenund Vermögensgemeinschaften (meistens als freie Lebensgemeinschaft bezeichnet) nicht zulässt, dieser gleichzeitig kein Hindernis für die Gewährung bestimmter Rechte für Personen darstellt, die in diesen Lebensgemeinschaften leben, soweit der Bereich dieser Rechte ihre Situation der rechtlichen Situation der Ehegatten nicht angleicht. In diesem Zusammenhang sieht die Situation der homosexuellen Personenund Vermögensgemeinschaften anders aus. Dieses bringt das Oberste Gericht in der Begründung einer Entscheidung zum Ausdruck: „Gegen die Erfassung der Gemeinschaften der Personen gleichen Geschlechts, die nach dem Vorbild der heterosexuellen Lebensgemeinschaften organisiert sind, mit dem Begriff der 'freien Lebensgemeinschaft' spricht die lange Tradition, auch die Sprachtradition. Wie in Lehrmeinungen zutreffend hervorgehoben wird, ist ein Gegenargument, dass man die Eigenschaften der freien Lebensgemeinschaften in Bezug auf das Modell der ehelichen Lebensgemeinschaften fasst, welche also die Lebensgemeinschaften der Personen verschiedenen Geschlechts betrifft“39. Dabei weist das Oberste Gericht darauf hin, „dass die eindeutige Unterscheidung der Beziehungen der Personen verschiedenen und gleichen Geschlechts im Großteil der Mitgliedstaaten besteht und in der Rechtsprechung des Gerichtshofs der Europäischen Union angenommen und berücksichtigt wird (Vgl. Entscheidung vom 17. Februar 1998 und Grant gegen South-West Trains Ltd, (C-249/96, Slg. 1998, I-621, Randnr. 45) und vom 31. Mai 2001, D und Königreich Schweden/Rat (C-122/99 P und C-125/99 P, Slg. 2001, I-4319)" 40.

Gleichzeitig ist in der Rechtsprechung der ordentlichen Gerichte und des Obersten Gerichts die gemeinsame Personenund Vermögensgemeinschaft der homosexuellen Paare bei Auflösung konkreter Vermögensprobleme, die mit einer Miete $u$. ä. verbunden sind, zulässig. Die Gerichte wenden jedoch in diesem Fall die Regulierung bezüglich der Ehe nicht an. Das hat das Oberste Gericht bezüglich der Vermögensberechnung zum Ausdruck gebracht. Das Oberste Gericht

\footnotetext{
${ }^{39}$ Fußnote 19.

40 Ebenda.
} 
hat festgestellt, dass „die Vermögensberechnungen nach der Beendigung der tatsächlichen Personen- und Vermögensgemeinschaft der Personen gleichen Geschlechts auf Grundlage der zum Inhalt der in dieser Beziehung gestalteten Verhältnisse entsprechenden Vorschriften des Zivilgesetzbuches erfolgen"41. Wiederum hat das Oberste Gericht bei der Entscheidung der Sache über die Miete festgestellt: „Die Person, die mit dem Mieter tatsächlich zusammenlebt, ist die Person, die mit dem Vermieter durch eine Gefühls-, Körperund Wirtschaftsbindung verbunden ist und auch die Person gleichen Geschlechts" 42 .

Die Gewährung bestimmter Rechte in der gerichtlichen Rechtsprechung oder in Gesetzen den heterosexuellen Paaren gegenüber (wovon in der Antwort auf die Frage 2 die Rede war), die in einer Personen- und Vermögensgemeinschaft zusammen sind, bei Nichtbeachtung von homosexuellen Paaren, die solch eine Gemeinschaft bilden, wäre mit dem Art. 31 der Verfassung, der das Verbot des Diskriminierung aus jedweden Gründen ausdrückt, unvereinbar, es sei denn, es gäbe wichtige Umstände, die sich auf die verfassungsmäßigen Werte stützen, die für die Erhaltung der begründeten Unterscheidung der hetero- und homosexuellen Paare sprächen.

Die Verfassung der Republik Polen lässt eine Institutionalisierung der Personen- und Vermögensgemeinschaft von zwei Personen nicht zu. Auf die Verfassung kann nur in einem begrenzten Umfang für die Begründung der Gewährung konkreter Rechte ausschließlich an heterosexuelle Lebensgemeinschaften, die in den Bereich der den Ehegatten vorbehaltenen Rechte nicht einschreiten, zurückgegriffen werden. Es geht um solche Situationen, in denen dafür die von der Verfassung geschützten Werte sprechen. In allen anderen Fällen spricht das im Art. 32 der Verfassung formulierte Verbot der Diskriminierung aus jedweden Gründen gegen die Gewährung von Rechten ausschließlich an heterosexuelle Lebensgemeinschaften.

\footnotetext{
41 Ebenda.

42 Bechluss (3) des Obersten Gerichts vom 28.12.2012, III CZP 65/12, Orzecznictwo Sądu Najwyższego - Izba Cywilna [Zeitschrift Rechtsprechung des Obersten Gerichts Zivilkammer; OSNC] 2013, Nr. 5, Pos. 57.
} 


\section{ISLAMISCHES RECHT}

In muslimischen Staaten sind die Menschen nicht nur nach Geschlecht, sondern auch nach der Religionszugehörigkeit klassifiziert. Es bestehen vier Hauptgruppen, die einen unterschiedlichen Rechtsstatus haben:

- Die Muslime, nur sie sind vollwertige Bürger und erlangen ihre Position durch die Geburt oder Konvertierung,

- Die Anhänger der monotheistischen Religionen, zu denen Juden, Christen und Samariter sowie Sabäer gehören,

- Die Anhänger der polytheistischen Religionen oder der durch den Islam nicht anerkannten Religionen: wie z.B. die Bahaiten, einen ähnlichen Rechtsstatus haben die Atheisten,

- Apostaten, das sind die Muslime, die sich vom Islam abgewandt haben, indem sie sich einer der genannten Gruppen angeschlossen haben. Die Apostaten sind in der schwierigsten Situation, weil besondere Rechtssanktionen gegen sie gerichtet sind.

Das islamische Recht basiert mithin auf drei fundamentalen Ungleichheiten: zwischen Muslimen und Nichtmuslimen, zwischen einem Mann und einer Frau und zwischen einem freien Menschen und einem Sklaven (heutzutage hat die dritte Unterscheidung an Bedeutung verloren). Rechtlich betrachtet erkennt die Scharia eine Überlegenheit eines Muslims über einen Nichtmuslim, ähnlich wie die Überlegenheit eines Mannes über eine Frau an. Die Frauen haben einen niedrigeren Status, dessen Quellen schon im Koran zu suchen sind: Sure 2, Vers 228 sieht vor: „Die Männer haben einen gewissen Vorrang vor ihnen"43. Beispielsweise weise ich auf die folgenden Unterschiede hin: nur ein Muslim - Mann kann eine Nichtmuslimin heiraten, nur ein Mann kann vier Ehefrauen haben, die Aussagen eines Mannes gleichen die Aussagen von zwei Frauen aus, die Muslimin kann nicht einen Nichtmuslim heiraten, die Frau erbt die Hälfte von dem, was der Mann erbt. Der Nichtmuslim kann nicht von einem Muslim erben.

43 Alle Zitate aus dem Koran zitiere ich nach: Koran, aus dem Arabischen übersetzt und mit einer Einführung und Fußnoten versehen von J. Bielawski, Warszawa: PIW 1986. Zuerst wird die Nummer der Sure und dann die Nummer des Ajat-Verses angegeben. 
$\mathrm{Da}$ der Islam eine Lebensweise ist, die die persönlichen, geistlichen, körperlichen und auch sozialen Aspekte des täglichen Lebens regelt, werden die Fragen des Familienrechts, darunter der Ehe, auch durch die Normen der Scharia rigoros festgelegt. In diesem Sinne ist hervorzuheben, dass die Anhänger des Islams großen Wert auf die Hervorhebung der Rolle und der Bedeutung der Familie und der Ehe im Leben von Muslimen legen, indem sie darstellen, dass die Ehe als Beziehung eines Mannes und einer Frau ein Fundament der ganzen Gesellschaft ist ${ }^{44}$.

In der muslimischen Gesellschaft begrenzt sich die Familie nicht auf die Kernfamilie (Eltern und Kinder), sondern sie umfasst auch: Großeltern, Onkel, Tanten, Cousins und Personen, die in die Familie durch die Ehe aufgenommen wurden. Die so verstandene große Familie hat die Aufgabe, die Kinder zu erziehen, die Minderjährigen vor negativen Außeneinflüssen zu schützen, die Religion weiterzuleiten, die Tradition, Bräuche und intime Seiten des Familienlebens $\mathrm{zu}$ pflegen. Trotz der Tatsache, dass der Einfluss der modernistischen Bewegungen viele muslimische Institutionen verletzt hat, blieb die Familie unberührt ${ }^{45}$.

Es kann nicht ausgeschlossen werden, dass eine der Ursachen dieser Erscheinung die Tatsache ist, dass obwohl das 20. Jahrhundert Reformversuche der Rechtssysteme in muslimischen Staaten ${ }^{46}$, insbesondere nach der für sie erlangten Unabhängigkeit gebracht hat, praktisch in allen Ländern, in denen der Islam herrscht, das einzige geltende Familienrecht die Scharia ist - das islamische Religionsrecht ${ }^{47}$, dessen Fundamente zwischen dem 7. und dem 10. Jahrhundert nach Christi entstanden sind. Die in den islamischen Ländern angenommenen Gesetze bezüglich des Familienrechts stimmen nicht nur überein, sondern greifen direkt auf die Scharia zurück.

\footnotetext{
44 Vgl. z.B. S. Abul A'ala Mawdudi, Islamic way of life, Online-Version: http://web.youngmuslims.ca/online_library/books/islamic_way_of_life/ [Zugang am 20.07.2013].

45 S. Hossein Nasr, Istota islamu. Trwałe wartości dla ludzkości [Der Kern des Islams. Beständige Werte für die Menschheit], Warszawa: Instytut Wydawniczy PAX 2010, S. 158.

46 Ein Anzeichen dafür ist die Übernahme der französischen, deutschen und englischen rechtlichen Muster, insbesondere im Bereich des Zivil- und Handelsrechts.

47 J.N.D. Anderson, Modern Trends in Islam Legal Reform and Modernisation in the Middle East, International and Comparative Law Quarterly 1971, Vol. 20, Nr. 1, S. 1.
} 
Die Muslime, anders als z.B. Buddhisten, stehen sehr kritisch zum Zölibat und betrachten die Ehe als rechts-religiöses Gebot. In dem oft zitierten Hadith hat Mohammed festgestellt: „Es gibt kein Zölibat im Islam“. Die Ehe ist die einzige berechtigte (anders ein Halal - genehmigte), legale Weise der Befriedigung des sexuellen Triebes zwischen einem Mann und einer Frau. Alle außerehelichen sexuellen Verhältnisse sind verboten und ein Ehebruch ist eine der schwersten Straftaten und unterliegt strengen Strafen.

Ein anderes Beispiel der Gleichheit zwischen Frauen und Männern ist die Bewilligung der Polygynie, das bedeutet eine Beziehung eines Mannes mit mehreren Frauen (maximal mit vier Frauen) ${ }^{48}$. Im Fall von Frauen ist die Scharia nicht so großzügig, denn sie verbietet völlig die Polyandrie, demnach die Beziehung einer Frau mit mindestens zwei Männern. Die Frage der Genehmigung der Polygamie der Männer und ihr Verbot bezüglich der Frauen verweist ausdrücklich auf die Nichtgleichberechtigung der Geschlechter im islamischen Recht. Sie basiert auf der Scharia und den Zivilrechtsgesetzen fast aller muslimischen Länder.

Das Familienrecht wird im islamischen Recht mit Hilfe einer Reihe von Institutionen, den „Rechten des persönlichen Status“, geregelt. Die sich mit dieser Problematik beschäftigenden Autoren sind davon überzeugt, dass dieser Rechtszweig außer dem Familienrecht auch andere Institutionen umfasst, insbesondere die Normen, die die Rechtsfähigkeit, Obhut, Vormundschaft, Erbnahme und die Errichtung des Testaments betreffen ${ }^{49}$.

48 Allerdings wurde die Polygynie in manchen Staaten verboten, z.B. in Tunesien und der Türkei. Vgl. Art. 112(1) of the Turkish Civil Code, 1926; and Art. 18 of the Tunician Code of Personal Status, 1956, in: R.K. Sinha, Muslim Law, Allahabad: Central Law Agency 2003, S. 39. Zu bedenken ist jedoch, dass die Polygynie zulässig aber nicht verpflichtend ist. Vgl. auch R. Sjukijajnen, der festgestellt hat, die Gesetze fast aller arabischen Ländern orientieren einen Muslim auf den Besitz von vier Ehegattinnen: Idem, Prawo muzutmańskie i ustawodawstwo rodzinne krajów Arabskiego Wschodu [Islamisches Recht und Familienrecht in den ostarabischen Ländern], [in:] L.R. Sûkiâjnen, V.E. Čirkin, Û.A. Ûdin, Prawo muzulmańskie (Struktura i podstawowe instytucje) [Islamisches Recht (Struktur und Grundinstitutionen)], Warszawa: Państwowe Wydawnictwo Naukowe 1990, S. 226.

49 Sjukijajnen, Fußnote 48, S. 210. 
Im Islam ist die Ehe, anders als z.B. im Hinduismus oder im Christentum, kein Sakrament ${ }^{50}$, sondern ein zivilrechtlicher Vertrag, der auf Grund der Parteivereinbarung abgeschlossen wird. Bemerkenswert ist, an dieser Stelle hinzuzufügen, dass da die Frau keine Vertragspartei ist, an der Gestaltung des Vertrages nicht teilnimmt und manchmal sogar für seinen Abschluss keine Genehmigung erteilen muss. Es reicht ihre stillschweigende Genehmigung oder ihre Zustimmung post factum aus.

Im islamischen Recht, ähnlich wie in anderen Rechtssystemen, kann man auch eine Reihe von Ausschlüssen finden, die es bestimmten Kategorien von Personen nicht erlauben, eine rechtswirksame Ehe zu schließen. Der Katalog dieser Hindernisse (Verbote) ist im Islam ziemlich breit und die Hauptlinie der Einteilung verläuft zwischen den dauerhaften Hindernissen, das bedeutet solchen, die infolge z.B. des Eintretens bestimmter rechtserheblicher Tatsachen nicht außer Kraft treten können und den zeitlichen Verboten, die nach dem Ablauf der durch das Recht bestimmten Zeit außer Kraft treten oder infolge des Eintretens bestimmter Ereignisse Rechtswirkungen entfalten. Der Sinn des zeitlichen Verbots läuft darauf hinaus, dass nach dem Wegfall der durch die Scharia genannten Hindernisse die Ehe zwischen Heiratswilligen wirksam geschlossen werden kann.

Mindestens einige der durch die Scharia ${ }^{51}$ genannten Ehehindernisse bestätigen eine privilegierte Rechtsstellung des Mannes gegenüber einer Frau, und manchmal brechen sie das Freiwilligkeitsprinzip der geschlossenen Ehe durch die Personen, die sich eine solche wünschen.

Eines dieser Hindernisse ist die Gleichberechtigung der Ehegatten und eigentlich die Gleichheit des Mannes im Vergleich zur Frau, die nach Meinung der Mehrzahl der muslimischen Juristen eine notwendige Voraussetzung des ehelichen Vertrages ist und seine Nichterfüllung stellt eine Grundlage für das Verlangen seiner Ungültigkeitserklärung durch die Parteien dar. Diese Regel hat

\footnotetext{
50 Der Islam kennt überhaupt keine Sakramente und aus diesem Grund braucht er keine Priester, die ihre Verwalter wären.

51 Die Scharia unterscheidet neben den oben genannten, folgende Eheverbote, die die rechtswirksame Eheschließung unmöglich machen: Verwandtschaft, Milchverwandtschaft, Verschwägerung, Alter, Geisteskrankheit oder Geistesschwäche, Gleichheit des Mannes gegenüber der Frau, die Religion.
} 
ihre Quelle in der Aussage vom Mohammed: „Bringt die Frauen nur mit ihnen Gleichstehenden unter die Haube". Die Nichterfüllung dieser Voraussetzung hat keinen Einfluss auf die Rechtskraft dieser Heiratsurkunde unter der Bedingung, dass die Parteien auf die Vollstreckung des "Gleichheitsrechts" verzichten, obwohl den Ehegatten die Pflicht der Eheauflösung durch das Gericht auferlegt werden kann. Davon ist unten die Rede.

Das Freiwilligkeitsprinzip beruht darauf, dass die Frau das Recht auf mindestens einen dem ihren gleichen sozialen Status des Ehemannes hat ${ }^{52}$. Der muslimische Mann kann natürlich erheblich höher in der gesellschaftlichen Hierarchie als seine Frau stehen. Die muslimischen Juristen erklären, dass sich dieses Prinzip hauptsächlich aus dem Charakter der muslimischen Familie ergibt, die sowohl patriarchalisch (auf dem Mann lastet die Pflicht des Familienunterhalts), patrilineal (die Herkunft erbt man in der männlichen Linie) als auch patrilokal (nach der Eheschließung zieht die Frau ins Haus ihres Mannes um) ist. In Syrien, Jordanien und Ägypten stellt die Nichterfüllung der Gleichheitsbedingung die Grundlage für das Verlangen der Ungültigkeitserklärung der Ehe nicht nur durch die Frau, sondern auch durch ihren Betreuer (Wali) dar und kann sogar durch die Verwandten der Frau initiiert werden. Solch eine Situation gab es im Jahre 2006 in Saudi-Arabien. Im Februar dieses Jahres haben die Polizisten das Haus von Fatima und Mansour al-Timani besucht, um Mansour die Ehescheidungsunterlagen auszuhändigen. Es hat sich erwiesen, dass die Scheidung auf Antrag der Verwandten von Fatima, die der Meinung waren, dass sie einen Mann "unter ihrem Niveau“

52 Einzelne Schulen der islamischen Rechts geben etwas andere Gleichheitskriterien an. Nach der Auffassung der Hanafitenschule bedingen die folgenden Faktoren die Gleichheit des Mannes gegenüber der Frau: Abstammung, Religion, Gleichheit (der Mann kann kein Sklave sein, wenn er eine freie Frau heiratet), der ausgeübte Beruf (er soll sich mit Handel beschäftigen oder ein Handwerker sein), der Grad der Frömmigkeit. Malikiten zählen wiederum zu den Gleichheitsbedingungen: Frömmigkeit, keinen körperlichen Defekt, Abstammung, der Vater des Ehemannes soll bekannt sein, der Ehemann darf kein Findelkind sein. Nach den Schafiiten sind diese Bedingungen: Position in der gesellschaftlichen Hierarchie, Freiheit von Defekten, Frömmigkeit, Beruf und Wohlstandsgrad. Die Hanbaliten weisen in dieser Materie auf die Frömmigkeit, Abstammung, den Wohlstandsgrad und den ausgeübten Beruf hin., Vgl. J.J. Nasir, The Islamic Law of Personal Status, London: Graham \& Trotman 1990, S. 59. 
geheiratet hat, durchgeführt worden ist. Obwohl die Ehegatten durch Gefühle miteinander verbunden waren und nicht vorhatten, sich scheiden $\mathrm{zu}$ lassen, ist die ganze Prozedur in Übereinstimmung mit der Rechtsordnung erfolgt. Fatima und ihr Mann haben eine Berufung beim König eingelegt, aber bis jetzt keine Antwort erhalten ${ }^{53}$.

Das mit der Religion verbundene eheliche Verbot privilegiert den Mann, der nach dem Koran keine Ehe mit einer Polytheistin, Atheistin oder Abtrünnigen schließen kann, aber die "Frauen des Buches", also eine Jüdin oder Christin, heiraten kann. Diese Begrenzung wurde ausdrücklich im Koran betont: „Heute sind euch die guten Dinge erlaubt. Und die Speise derjenigen, denen die Schrift gegeben wurde, ist euch erlaubt, und eure Speise ist ihnen erlaubt. Und die Ehrbaren von den gläubigen Frauen und die ehrbaren Frauen von denjenigen, denen vor euch die Schrift gegeben wurde ${ }^{\prime 54}$. Dies ist insofern bemerkenswert, da nach der Trauung die Frau, die nicht Muslimin ist, keine gleichen Rechte wie die EhefrauMuslimin in Anspruch nimmt. Die Ehefrau-Nichtmuslimin, die nach der Vermählung nicht zum Islam übergetreten ist, hat kein Recht, von ihrem Ehemann zu erben. Sie kann nur auf Grund des Testaments nicht mehr als 1/3 des Vermögens ihres Mannes erhalten. Im Falle der Scheidung hat sie wiederum kein Recht auf die Sorge für Kinder.

Dagegen verbietet die Scharia, dass eine Muslimin von einem Nichtmuslim geheiratet wird. Das islamische Recht stellt ausdrücklich fest, dass die zwischen einer Muslimin und einem Nichtmuslim geschlossene Ehe nichtig und wirkungslos in einem muslimischen Land ist, wenn sie auch im Land ihrer Schließung wirksam wäre ${ }^{55}$. In einem muslimischen Land wird eine solche Ehe durch eine dafür zuständige Behörde nicht registriert und die Frau, die diese Tat verübt hat, begeht eine Sünde/eine Straftat des Ehebruchs.

Von den drei zeitlichen Ehehindernissen: eine bestehende Ehe, die Zeit der Iddat, der Glaubensunterschied, betrifft das zweite - Iddat -

\footnotetext{
53 N. Darwish, Okrucieństwo w majestacie prawa. Prześladowanie kobiet w świecie islamu [Grausamkeit unter der Hoheit des Rechts. Verfolgung von Frauen in der islamischen Welt], Warszawa: Kefas 2011, S. 79-80.

54 Koran, 5, 5.

55 S.A.A. al-Maududi, The Rights and Duties of Spouses, Delhi: Markazi Maktaba Islami Publishers 2000, S. 19.
} 
ausschließlich die Frauen. Der Begriff "Iddat" bedeutet die Zeit, in der die Frau, deren Ehe durch die Scheidung, den Tod ihres Ehemannes oder durch die Todeserklärung aufgelöst wurde, verpflichtet ist, in Abgeschiedenheit zu leben und keinen anderen Mann zu heiraten. Die muslimischen Juristen beweisen, dass das genannte Ehehindernis das Ziel hat, den Vater des eventuellen Kindes eindeutig zu benennen ${ }^{56}$.

Im Islam stellt die Ehe einen Vertrag dar. Somit besteht er, wie ein jeder Vertrag, aus Bestandteilen, die als unerlässlich für sein Bestehen gelten. Diese Elemente werden mit dem Begriff "rukn" (Säulen, Plural - arkan) bestimmt und sind die obligatorischen Elemente jedes ehelichen Vertrages. Neben den Säulen des ehelichen Vertrages bestehen auch "szart" (Plural: szurut), die als "Vorbedingungen" übersetzt werden können, die für einen wirksamen ehelichen Vertrag und die Bewirkung bestimmter Rechtsfolgen unerlässlich sind ${ }^{57}$. Da bestimmte Schulen des islamischen Rechts nicht im Stande sind, eindeutig "arkan" und "szurut" zu bestimmen, wird hier von ausführlichen Erwägungen $\mathrm{zu}$ dieser Materie abgesehen. Die muslimischen Juristen sind damit einverstanden, dass zu den "arkan" des ehelichen Vertrages "Angebot und seine Annahme" (Al-Ijaab wa al-qubul) gehören ${ }^{58}$. Die Mehrzahl der Wissenschaftler sind sich darüber einig, dass auch die Vertragsparteien: der zukünftige Ehemann und der Betreuer der Frau zu den „arkan“ des Vertrages gehören. Einige zählen zu den "arkan", aber die Mehrheit zu "szurut", folgende Elemente des ehelichen Vertrages: Anwesenheit von Zeugen, "Mahr" und Vertragsinhalt ${ }^{59}$.

\footnotetext{
56 Im Falle der widerrufenen Scheidung beträgt die Iddat 3 Monate. Der Mann kann sie widerrufen und die Ehe wird dann nicht aufgelöst. Ist der Ehemann verstorben oder wird er wegen Verschollenheit durch das Gericht für tot erklärt, beträgt die Wartezeit 4 Monate und 10 Tage, Vgl. Koran, II-234.

$57 \mathrm{Zu}$ diesem Thema: Vgl. S. W. Witkowski, Wprowadzenie do prawa muzutmańskiego. Wybrane instytucje [Einfuhrung ins musulmanischen Recht. Ausgewalte Institutionen], Warszawa: Wydawnictwo Naukowe Scholar, S. 92-98, und M. Zyzik, Matżeństwo w prawie muzutmańskim, (Ehe ins musulmanischen Recht] Warszawa: Dom Wydawniczy Elipsa 2003, S. 104-106.

58 So auch J.J. Nasir, The Status of Women Under Islamic Law and Modern Islamic Legislation, Leiden, Boston: Brill 2009, S. 60.

59 H.A. Jawad, The Rights of Women in Islam. An Authentic Approach, New York: Macmillan Press 1998, S. 32-33.
} 
Manche der Bestandteile des ehelichen Vertrages heben ausdrücklich die privilegierte Rechtsstellung des Mannes gegenüber der Frau hervor. Um eine Ehe nach dem islamischen Recht zu schließen, ist notwendig:

- ein ehelicher Vertrag,

- die Zustimmung der beiden Vertragsparteien,

- Mahr - d. h. ein Geschenk des Ehemannes für die Braut (ohne Geschenk ist die Ehe nichtig),

- Gleichheit des Mannes gegenüber der Frau,

- mindestens zwei männliche Zeugen,

- öffentliche Bekanntmachung der Eheschließung,

- Anwesenheit des offiziellen Faktors,

- „Wali" - der Betreuer der Frau (in manchen Fällen ist das auch der Betreuer des Mannes),

- dass der eheliche Vertrag nicht von der Frau ausgefertigt wird.

Der eheliche Vertrag bestimmt die Rechte und Pflichten der Parteien und im islamischen Recht ist das nicht nur der Vertrag zwischen den Heiratswilligen, sondern auch zwischen zwei Familien. Die muslimische Ehe wird auf Grund der Vereinbarung zwischen dem Bräutigam und dem "Wali“ - dem Betreuer der Frau geschlossen. Drei Schulen des islamischen Rechts vertreten übereinstimmend den Standpunkt, dass die Frau an der Ausfertigung des ehelichen Vertrags nicht teilnehmen soll. Nur die Hanafitenschule meint, dass eine reife, psychisch gesunde Frau ohne irgendeine Zustimmung die Ehe schließen kann ${ }^{60}$. Sowohl die Darstellung des Angebots als auch seine Annahme sollten während einer Sitzung stattfinden.

\footnotetext{
60 Zyzik, Fußnote 57, S. 92. Dies ist insofern bemerkenswert, als die Scharia ausdrücklich auf den Unterschied zwischen der Jungfrau und der Nichtjungfrau hindeutet, gegenüber denen jeweils andere Regulationen bezüglich der Rolle des Betreuers - "Wali" - bei der Eheschließung Anwendung finden. Auf kuriose Weise erklärt diese Frage M. Zyzik, indem er überzeugt ist, dass die Nichtjungfrau als Frau, die mehr Erfahrung in Kontakten mit Männern hat, selbst über die Eheschließung entscheiden kann und der Betreuer ihr lediglich bei der Vertragsgestaltung helfen soll. Die Jungfrau bedarf dagegen erheblich mehr Hilfe seitens ihres Betreuers, Zyzik, Fußnote 57, S. 93. Es muss dabei geklärt werden, dass die "legale“ Nichtjungfrau im Lichte der Scharia ausschließlich die Witwe oder geschiedene Frau sein kann. Somit ist schwer anzuerkennen, dass z.B. die 30-jährige Jungfrau weniger Lebenserfahrung hat als die geschiedene 18-jährige Frau, die keine Jungfrau ist.
} 
Ein ehelicher Vertrag kann von Seiten der Muslimin, genauer gesagt von Seiten ihres Betreuers, eine Reihe von Bedingungen enthalten, z.B. das Recht auf die Verweigerung der Genehmigung für ihren Ehemann, eine Ehe mit einer anderen Frau zu schließen, den Rechtsvorbehalt, eine Arbeit anzunehmen, eine Ausbildung fortzusetzen u.ä. Während der Ehe soll sich die Muslimin ihrem Ehemann unterstellen, sich um den Haushalt kümmern und alle sexuellen Bedürfnisse ihres Mannes erfüllen sowie ihm gehorsam sein. Die Konstruktion des ehelichen Vertrags privilegiert eindeutig den Ehemann gegenüber der Ehefrau.

Auch die Rechtsvorschriften von vielen islamischen Ländern genehmigen im ehelichen Vertrag verschiedene Bedingungen nach dem Willen der den Vertrag abschließenden Parteien. Dabei ist erforderlich, dass diese Bedingungen möglichst $\mathrm{zu}$ erfüllen sind, mit dem Zweck der Ehe übereinstimmen und nicht im Widerspruch zur Scharia stehen. Die dem islamischen Recht entgegenstehenden Bedingungen haben keine Nichtigkeit der Ehe zur Folge, aber sie sind als ungültig zu betrachten. Die Nichteinhaltung der vom Ehemann durch das Recht erlaubten und im Vertrag enthaltenen Bedingungen gibt der Ehefrau die Gelegenheit, beim Gericht einen Anspruch auf Eheauflösung zu erheben.

In vielen Ländern genehmigt das Familienrecht der Ehefrau den Bedingungsvorbehalt, der ihr ermöglicht, bei einer neuen Eheschließung von ihrem Ehemann eine Scheidung zu erreichen ${ }^{61}$.

In diesem Zusammenhang sei daran erinnert, dass die Rechtsvorschriften in fast allen islamischen Ländern eine traditionelle Norm der Scharia enthalten, die den Besitz von vier Ehefrauen genehmigt, und es z.B. in Jordanien keine Begrenzung der Polygamie in diesem Bereich (4 Ehefrauen) gibt. Eine einzige Beschränkung für die Polygamie ist nach der Scharia die Bedingung gerechter Behandlung aller Ehefrauen ${ }^{62}$. Dies ist insofern bemerkenswert, als der Koran sogar davon überzeugt ist, dass diese Bedingung zur Realisierung unmöglich ist:

61 Sjukijajnen, Fußnote 48, S. 225.

62 Eine solche Bedingung sehen z.B. die Rechtsvorschriften in Jemen vor, ebenda, S. 226. 
„Und ihr könnt zwischen den Frauen keine Gerechtigkeit üben, so sehr ihr es auch wünschen mögt" 63 .

Es bedarf manchmal der Zustimmung des Gerichts für die weiteren Ehefrauen (Syrien, Marokko). Vor dem Abschluss eines ehelichen Vertrages mit einer weiteren Frau ist ihr über die schon bestehenden Ehen Mitteilung zu machen. Die erste Ehefrau, selbst wenn sie sich im ehelichen Vertrag nicht eine Zustimmung auf eine weitere Ehefrau vorbehalten hat, kann beim Gericht eine Scheidungsklage einlegen, indem sie sich auf den ihr wegen der von ihrem Ehemann geschlossenen Ehe zugefügten Schaden bezieht.

Der eheliche Vertrag soll für die Vertragsparteien verständlich sein. Es ist nicht notwendig, dass seine Schlüsselbegriffe (z.B. "nikah" als Synonym der Ehe) im Arabischen ausgedrückt werden. Die Heiratswilligen sollten die Zustimmung zu der Eheschließung erteilen $^{64}$ und an dieser Handlung soll der Betreuer der Frau teilnehmen. Die Braut muss nicht einmal bei ihrer eigenen Trauung anwesend sein, es reicht ihre geleistete Unterschrift unter dem Vertrag65.

Der Betreuer (Wali) soll eine volljährige, geistig gesunde Person sein. Er muss ein Muslim sein und er ist fast immer ein naher Verwandter der Braut. Der „Wali“ der Christin kann ihr männlicher Verwandter sein. Wenn die Frau oder der Mann keine Verwandten haben, die im Lichte des Rechts die Pflichten des Betreuers erfüllen können, übernimmt das Gericht seine Rolle (so die Rechtsvorschriften in Jemen, Syrien und Jordanien). In einigen Fällen kann "Wali" auch der Betreuer des Bräutigams sein ${ }^{66}$.

63 Koran, 4, 129, Ibn Warraq ist überzeugt, dass sich die Bedingung der gerechten Behandlung auf die gleiche Bedenkung der Ehefrauen mit Geschenken beschränkt, sie aber mit Liebe oder Sex nichts zu tun hat. Ebenda, S. 398.

64 Die Zustimmung kann von der Jungfrau stillschweigend ausgedrückt werden. Berichtet von Abu Huraira, Vgl. Sahih al-Buchari, Buch 62, 1-67, Wedlock, Marriage (Nikah), OnlineVersion:

http:/ / www.searchtruth.com/book_display.php?book=62\&translator=1\&start=19\&number $=18$ [Zugang am 12.07.2013].

65 E. Khidayer, Życie po arabsku [Das Leben auf Arabiach], Warszawa: Prószyński i S-ka 2013, S. 83.

66 Sjukijajnen, Fußnote 48, S. 220. Die Gesetzgebung des Jemen genehmigt dem Betreuer eines Minderjährigen (Jungen), dass er ihn sogar gegen seinen Willen verheiraten kann. Ebenda, S. 221. 
Nach der islamischen Scharia ist die Trauung gültig, wenn während ihrer Schließung mindestens zwei männliche muslimische Zeugen anwesend sind. In der Theorie genehmigt das islamische Recht die Anwesenheit von weiblichen Zeugen, aber nur in der Situation, wenn Männer fehlen, die diese Rolle wahrnehmen. In der Praxis treten in dieser Stellung ausschließlich Männer auf67.

Die heutigen Rechtsvorschriften der muslimischen Länder verlangen für die Gültigkeit der Ehe eine Heiratsurkunde und die Anwesenheit des offiziellen Faktors (der offiziellen die Ehe schließenden Person). Ein Beamter, der Hochzeitspaare traut, wird in Ägypten als "Mazun" (anders - mad hun) bezeichnet. Zu seiner Zuständigkeit gehört auch die Registrierung der Scheidung 68 .

Eine Besonderheit des islamischen Familienrechts, die einen erheblichen Einfluss auf die Gültigkeit des abgeschlossenen Aktes hat, ist "mahr" (anders - sadaqa) - die Brautgabe des Ehemannes für die zukünftige Ehefrau. Im islamischen Familienrecht dient dieser Begriff zur Beschreibung der Bezahlung, die die Ehefrau von ihrem Ehemann für den Sex erhältt69. J. L. Esposito ist wiederum der Ansicht, dass die Bezahlung der Ehefrau durch den Ehemann für die Ehe zusteht ${ }^{70}$. Die genannte Konstruktion "Mahr" deutet sowohl im ersten als auch im zweiten Fall ausdrücklich auf die sachliche und nicht persönliche Behandlung der Frau hin.

"Mahr" ist meistens zweigeteilt: der erste Teil wird während der Ehe ausgezahlt und wird als sofortiger "Mahr" (muajjal) bezeichnet, und das, was der Ehefrau erst nach der Scheidung oder dem Tod oder

\footnotetext{
67 Khidayer, Fußnote 65, S. 85-86. Nach der Scharia können die Zeugen auch ein Mann und zwei Frauen sein, Nasir, Fußnote 58, S. 62.

68 Dieser Beamte wird manchmal als Religionsnotar bezeichnet. Vgl. M. Berger, N. Sonneveld, Sharia and National Law in Egypt, [in:] J.M. Otto (Red.), Sharia and National Law in the Muslim World: First Global Overview from Saudi Arabia to Indonesia, Leiden-Manchester: Amsterdam University Press 2010, S. 75.

69 So N. Darwish und dort angeführte Auffassung der muslimischen Juristen, der Klassiker der Scharia, Vgl. ebenda, S. 75-76.

70 L. Esposito, N.J. DeLong-Bas, Women in Muslim Family Law, Syracuse: Syracuse University Press, 2001, S. 23.
} 
durch ein anderes im Vertrag vereinbartes Geschehen übergeben wird, wird rechtlich als ein späterer "Mahr" (muwajjal) bezeichnet ${ }^{71}$.

"Mahr" kann sowohl in Sachleistung als auch in bar gezahlt werden. Seine Unter- und Obergrenze wird durch das Recht nicht eindeutig festgelegt, obwohl im ehelichen Vertrag die Höhe des "Mahr" genau festgelegt werden muss. Die Bestimmungen bezüglich des "Mahr" werden vom Bräutigam, seiner Familie und vom Betreuer der Braut ausgehandelt ${ }^{72}$.

Die muslimischen Juristen weisen darauf hin, dass die Brautgabe alles sein kann, was einen Wert hat und nicht Scharia-widrig ist. Zum Beispiel können teure Weine keine Gabe für die Ehefrau sein, weil Alkohol für Muslime "haram" - im Lichte der Scharia verboten ist. Nach der Scharia stellt "Mahr" das Eigentum der Ehefrau dar und sie entscheidet ausschließlich über seine Bestimmung. "Mahr" wird der Ehefrau nach dem Abschluss des ehelichen Vertrags oder nach dem Vollzug der Ehe ausgezahlt. Indem auf ausführliche Erwägungen bezüglich der rechtlichen Aspekte von "Mahr" in der Scharia verzichtet wird, möchte ich betonen, dass er eine Art der sozialen Sicherheit für die Ehefrau darstellen kann, denn der islamische Ehemann kann sich von dieser jederzeit scheiden lassen ${ }^{73}$, und sie ohne Existenzmittel zurücklassen.

Eine der wichtigsten Institutionen des Familienrechts der arabischen Länder ist die Ehescheidung. Das Recht der meisten von ihnen weist auf mehrere Scheidungsursachen hin. Die wichtigsten sind: Scheidung auf Initiative des Ehemannes, die Auflösung der Ehe nach der entsprechenden Vereinbarung der Ehegatten, Auflösung des Ehebandes auf Grundlage einer Gerichtsentscheidung.

71 So P. Fournier, Flirting with God in Western Secular Courts: Mahr in the West, International Journal of Law, Policy and the Family 2010, Vol. 24, Nr. 1, S. 69. M. Zyzik unterscheidet wiederum einen bestimmten "Mahr" (mahr musamma) und einen grundlegenden "Mahr" (mahr al-misal). Der erste "Mahr" ist eine Gabe, deren Höhe durch die Parteien des ehelichen Vertrages vereinbart wurde. Der grundlegende "Mahr" ist dann eine Gabe, deren Höhe von der gesellschaftlichen Position der Frau abhängig ist, Vgl. ebenda, S. 70. Als Kriterien für die Festlegung der Position der Frau dienen: Alter, Anmut, Status der weiblichen Verwandten seitens des Vaters, Wohlstandsgrad und Frömmigkeit und Jungfräulichkeit der Verlobten, ebenda.

72 L. Blenkhorn, Islamic Marriage Contracts in American Courts: Interpreting Mahr Agreement as Prenuptials and Their Effect on Muslim Women, Southern California Law Review 2002, Vol. 76 Nr. 1, S. 197, Online-Version: http://lawweb.usc.edu/why/students/orgs /lawreview/documents/Blenkhorn_Lindsey_76_1.pdf [Zugang am 10.09.2011].

73 So auch Khidayer, Fußnote 65, S. 86. 
Bemerkenswert ist auch, dass die Umsetzung der Scharianormen in die Rechtsvorschriften aller arabischen Länder die Scheidung für eine fundamentale Form der Eheauflösung hält und in dieser Materie hat der Ehemann fast unbeschränkte Rechte.

Die Scheidung durch "talaq"74 beruht darauf, dass der Ehemann nach der Scharia selbst eine sofortige und unwiderrufliche Scheidung durch das dreimalige Aussprechen des Wortes "talaq": "du bist geschieden", durchführen kann ${ }^{75}$. Wenn der Ehemann zu seiner Ehefrau "talaq" (du bist geschieden) sagt, und wenn er in einem Iddat-Zeitraum seine Worte nicht widerrufen hat, wird die Ehe aufgelöst. Wird vom Ehemann nur einmal oder auch zweimal das Wort "talaq" gesprochen, haben wir es mit einer widerrufenen Scheidung zu tun. Dies erlegt der Ehefrau die Pflicht auf, im Iddat-Zeitraum bei ihrem Ehemann zu wohnen. In dieser Zeit kann der Ehemann die Worte „ich nehme dich zurück" aussprechen und die Ehe liegt weiter vor.

Die durch die Scharia erarbeitete Scheidungsprozedur auf Initiative des Ehemannes wird auch gegenwärtig angewendet. Beispielsweise kann darauf hingewiesen werden, dass die derzeitigen Rechtsvorschriften vieler muslimischer Länder ${ }^{76}$ direkt vorsehen: der volljährige Ehemann, der über die Geschäfts- und Handlungsfähigkeit verfügt, kann sich jederzeit nach seinem Willen von der Ehefrau scheiden lassen, ohne einen Grund $\mathrm{zu}$ nennen. Der Ehefrau steht dieses Recht nicht $\mathrm{zu}$ und die Prozedur, ihrerseits die Scheidung $\mathrm{zu}$ erreichen, ist sehr schwer durchzuführen und mit hohen Kosten verbunden ${ }^{77}$. Die Fragen bezüglich der Eheauflösung weisen im islamischen Recht eindeutig auf die ungleiche rechtliche Lage der Ehegatten hin, denn das Recht auf eine einseitige Scheidung, ohne eine Begründung anzugeben, steht ausschließlich dem Ehemann zu.

\footnotetext{
74 Dieses arabische Wort bedeutet "befreien“ oder "laufen lassen".

75 Die muslimischen Juristen halten heutzutage die Auflösung des Ehebandes im Wege der „Talaq“ durch die Sendung einer E-Mail oder einer SMS für zulässig, ebenda, S. 78.

76 Die Talaq-Prozedur findet z.B. in Ägypten, Pakistan, Afghanistan, Saudi-Arabien und in vielen anderen muslimischen Ländern Anwendung.

77 Mehr dazu L. Welchmann, Egypt: New Deal on Divorce, International Survey of Family Law 2004, S. 123-141. Vgl. auch Berger, Sonneveld, Fußnote 68, S. 75.
} 
Bezüglich der Problematik der Lebenspartnerschaften oder der freien Lebensgemeinschaft ist anfangs festzustellen, dass diese Fragen durch das islamische Recht überhaupt nicht geregelt sind. Die Scharia lässt nämlich nicht nur keine außerehelichen Sexualbeziehungen $\mathrm{zu}$, sondern verbietet auch Personen verschiedenen Geschlechts unter einem Dach zu wohnen, es sei denn diese Personen sind nah miteinander verwandt. Wie ich schon oben nachgewiesen habe, sind die außerehelichen Sexualbeziehungen nicht nur Sünden, sondern sie gehören zu den besonders schweren Straftaten der Hudud-Kategorie.

Eine ähnliche Situation tritt im Verhältnis $\mathrm{zu}$ Beziehungen der Personen gleichen Geschlechts auf. In der muslimischen Gesellschaft hält man die Homosexualität für ein perverses Verhalten oder das Symptom einer psychischen Krankheit. In diesem Zusammenhang hält man die Homosexuellen für depravierte und wahnsinnige Personen und eine solche Auffassung hat eine Strafe oder im besten Fall eine Therapie zur Folge ${ }^{78}$. Das Gesetzesrecht der islamischen Länder sieht schwere Strafen für homosexuelle Beziehungen vor, z.B. in Saudi-Arabien droht für diese Straftat die Todesstrafe ${ }^{79}$. Somit sind die Beziehungen von Personen gleichen Geschlechts im islamischen Recht illegal.

In aller Kürze kann man sagen, dass die Frage der Legalisierung von nichtehelichen Lebensgemeinschaften der Personen verschiedenen Geschlechts und mehr noch der Beziehungen der Personen gleichen Geschlechts im offiziellen, öffentlichen Diskurs in muslimischen Ländern überhaupt nicht vorliegt.

Die Scharia - das islamische Recht - sieht vor, dass die aus einem Mann und einer Frau bestehende Familie die Grundlage der Gesellschaft bildet, weil die Ehe die einzige berechtigte (anders "halal" - durch

\footnotetext{
78 Vgl. M. Woźniak, Społeczność LGTB i ich organizacje na Bliskim Wschodzie [Die LGTB-Gesellschaft und ihre Organisationen im Nahen Osten], [in:] K. Górak-Sosnowska (Red.), Queer a islam. Alternatywna seksualność w kulturach muzutmańskich [Queer und Islam. Alternative Sexualität in der muslimischen Kultur], Sopot: Smak Słowa 2012, S. 202.

79 Ebenda, S. 212.
} 
das Recht erlaubte), legale Art der Befriedigung des sexuellen Triebes zwischen Mann und Frau ist. Dieses Recht beschäftigt sich nicht nur nicht mit nichtehelichen Lebensgemeinschaften, sondern es verbietet sie direkt.

Das Wesen des islamischen Familienrechts ist eine vollständige Kontrolle und Regulierung des Familienlebens. Dieses Ziel schwebt auch dem ehelichen Vertrag vor, der die Rechte und Pflichten der Parteien bestimmt, von denen nur eine (der Mann) an den Vertragsverhandlungen teilnimmt. Das betrifft nicht nur den Vertrag zwischen den Heiratswilligen, sondern auch den Vertrag zwischen zwei Familien. Das islamische Familienrecht als Teil der Scharia sanktioniert die rechtliche Ungleichheit der Parteien, die ihm unterliegen.

Dieses Recht festigt unter Bezugnahme auf die religiösen Quellen die privilegierte Position des Mannes gegenüber der Frau (Überlegenheit des Ehemannes gegenüber der Ehefrau), indem es dem Ehemann gestattet und der Frau verweigert, Rechtshandlungen vorzunehmen (z.B. das Recht vier Ehefrauen $\mathrm{zu}$ haben, das Talaq-Recht - einseitige Scheidung auf Initiative des Ehemannes, ohne einen Grund zu nennen und ohne rechtliche Prozedur durchzuführen). In einer besonders schwierigen Rechtslage befinden sich Frauen, die Muslime geheiratet haben und nicht zum Islam übergetreten sind. Im Falle einer fehlenden Eintragung im Testament können die Frauen von ihren verstorbenen Ehemännern nicht erben, und es steht ihnen auch kein Sorgerecht für die Kinder zu. 
